\title{
Global Behavior of Solutions in a Predator-Prey Cross-Diffusion Model with Cannibalism
}

\author{
Meijun Chen, Shengmao Fu $(\mathbb{D}$, and Xiaoli Yang \\ College of Mathematics and Statistics, Northwest Normal University, Lanzhou 730070, China \\ Correspondence should be addressed to Shengmao Fu; fusm@nwnu.edu.cn
}

Received 20 February 2020; Accepted 7 May 2020; Published 22 May 2020

Academic Editor: Lucia Valentina Gambuzza

Copyright (C) 2020 Meijun Chen et al. This is an open access article distributed under the Creative Commons Attribution License, which permits unrestricted use, distribution, and reproduction in any medium, provided the original work is properly cited.

The global asymptotic behavior of solutions in a cross-diffusive predator-prey model with cannibalism is studied in this paper. Firstly, the local stability of nonnegative equilibria for the weakly coupled reaction-diffusion model and strongly coupled crossdiffusion model is discussed. It is shown that the equilibria have the same stability properties for the corresponding ODE model and semilinear reaction-diffusion model, but under suitable conditions on reaction coefficients, cross-diffusion-driven Turing instability occurs. Secondly, the uniform boundedness and the global existence of solutions for the model with SKT-type crossdiffusion are investigated when the space dimension is one. Finally, the global stability of the positive equilibrium is established by constructing a Lyapunov function. The result indicates that, under certain conditions on reaction coefficients, the model has no nonconstant positive steady state if the diffusion matrix is positive definite and the self-diffusion coefficients are large enough.

\section{Introduction}

In 1999, Magnússon [1] proposed the following predatorprey model:

$$
\left\{\begin{array}{l}
\frac{\mathrm{d} X}{\mathrm{~d} t}=-M_{\mathrm{mat}} X+A Y+B X Y+C X Z, \\
\frac{\mathrm{d} Y}{\mathrm{~d} t}=R X-A Y-M_{\mathrm{juv}} Y-S X Y, \\
\frac{\mathrm{d} Z}{\mathrm{~d} t}=T Z-U Z^{2}-V X Z,
\end{array}\right.
$$

where $X, Y$, and $Z$ represent the biomasses of adult predators, juvenile predators, and prey, respectively, $M_{\text {mat }}$ and $M_{\text {juv }}$ represent the death rates of adult predators and juvenile predators, respectively, $A$ denotes the specific rate of juveniles recruited to the adults class, $R$ denotes the birth rate, $T$ and $U$ are logistic coefficients, $S$ represents the cannibalism attack rate, $B=\gamma S$, where $\gamma$ is the conversion efficiency of eaten juveniles into adult biomass, and the biological meanings of $V$ and $C$ are similar to $S$ and $B$. For more details on model (1), please refer to [1].
Let

$$
\begin{aligned}
& u=\frac{S}{M_{\text {mat }}} X, \\
& v=\frac{S A}{M_{\text {mat }}^{2}} Y, \\
& w=\frac{C}{M_{\text {mat }}} Z, \\
& \tilde{t}=M_{\text {mat }} t,
\end{aligned}
$$

and still denote $\tilde{t}$ by $t$; then, (1) becomes

$$
\left\{\begin{array}{l}
\frac{\mathrm{d} u}{\mathrm{~d} t}=-u+v+a u v+u w \\
\frac{\mathrm{d} v}{\mathrm{~d} t}=b u-g v-u v \\
\frac{\mathrm{d} w}{\mathrm{~d} t}=s w-r w^{2}-e u w
\end{array}\right.
$$


where

$$
\begin{aligned}
& a=\frac{B M_{\mathrm{mat}}}{S A}=\frac{\gamma M_{\mathrm{mat}}}{A}, \\
& b=\frac{R A}{M_{\mathrm{mat}}^{2}}, \\
& g=\frac{A+M_{\mathrm{juv}}}{M_{\mathrm{mat}}}, \\
& s=\frac{T}{M_{\mathrm{mat}}}, \\
& r=\frac{U}{C}, \\
& e=\frac{V}{S} .
\end{aligned}
$$

We can see that $e$ is the only parameter depending on $S$ in system (3).

Most works on cannibalism adopt a McKendrick-von Foerster age-structured model [2-4], but the model is usually rewritten as an ODE system or nonlinear Volterra integral equations $[4,5]$. In 1995, Kohlmeier and Ebenhöh [6] studied a two-dimensional ODE model without any structure, and they obtained that, in some cases, cannibalism can lead to a higher long term predator stock size. Then, van den Bosch and Gabriel [7] found that cannibalism can stabilize a predator-prey system in a structured model where the oscillations are due to age structure.

Magnússon [1] discussed model (1) which is in some ways a simplification of the system studied by van den Bosch and Gabriel in [7]. Magnússon made two simplifications. Firstly, it is assumed that all juveniles are vulnerable to predation by the adults. Secondly, instantaneous maturation into the adult class is proportional to the present juvenile biomass, i.e., a constant per capita rate of maturation, and making this simplifying assumption means that any oscillations that may occur are not caused by a delay inherent in the system. Moreover, the stability and Hopf bifurcation of solutions for system (3) were studied in [1]. The author obtained the following conclusions. On one hand, if the mortality rate of juveniles is low and/or the recruitment rate to the mature population is high, then there is a stable equilibrium with all three population sizes as positive. On the other hand, if the mortality rate of juveniles is high and/ or the recruitment rate to the mature population is low, then the equilibrium will be stable for low levels of cannibalism, but a loss of stability by a Hopf bifurcation will take place as the level of cannibalism increases.

As we all know, the growth of biological population depends not only on time, but also on spatial distribution. Spatial species interaction includes the self-diffusion, which is the natural dispersive force of movement of individual, and the cross-diffusion, that is, the population fluxes of one species due to the presence of other species [8-16]. Therefore, taking into account the effect of competition for resources on species growth law, we are naturally led to the following weakly coupled reaction-diffusion model:

$$
\left\{\begin{array}{l}
u_{t}=d_{1} \Delta u-u+v+a u v+u w, \quad x \in \Omega, t>0 \\
v_{t}=d_{2} \Delta v+b u-g v-u v, \quad x \in \Omega, t>0 \\
w_{t}=d_{3} \Delta w+s w-r w^{2}-e u w, \quad x \in \Omega, t>0 \\
\partial_{\eta} u=\partial_{\eta} v=\partial_{\eta} w=0, \quad x \in \partial \Omega, t>0 \\
u(x, 0)=u_{0}(x) \\
v(x, 0)=v_{0}(x) \\
w(x, 0)=w_{0}(x) \\
x \in \Omega
\end{array}\right.
$$

and the Shigesada-Kawasaki-Teramoto model with strongly coupled cross-diffusion

$$
\left\{\begin{array}{l}
u_{t}=\Delta\left(d_{1} u+\alpha_{11} u^{2}+\alpha_{12} u v+\alpha_{13} u w\right)-u+v+a u v+u w, \quad x \in \Omega, t>0, \\
v_{t}=\Delta\left(d_{2} v+\alpha_{21} u v+\alpha_{22} v^{2}+\alpha_{23} v w\right)+b u-g v-u v, \quad x \in \Omega, t>0, \\
w_{t}=\Delta\left(d_{3} w+\alpha_{31} u w+\alpha_{32} v w+\alpha_{33} w^{2}\right)+s w-r w^{2}-e u w, \quad x \in \Omega, t>0, \\
\partial_{\eta} u=\partial_{\eta} v=\partial_{\eta} w=0, \quad x \in \partial \Omega, t>0, \\
u(x, 0)=u_{0}(x), \\
v(x, 0)=v_{0}(x), \\
w(x, 0)=w_{0}(x), \\
x \in \Omega
\end{array}\right.
$$

where $\Omega \subset \mathbb{R}^{n}$ is a bounded domain with smooth boundary $\partial \Omega$ and $\eta$ is the outward unit normal vector of the boundary $\partial \Omega, \partial_{\eta}=\partial / \partial \eta$. The coefficients $d_{i}(i=1,2,3)$ are the diffusion rates of $u, v$, and $w, \alpha_{i i}(i=1,2,3)$ are referred as selfdiffusion pressures, and $\alpha_{i j}(i \neq j, i, j=1,2,3)$ are crossdiffusion coefficients which may be positive, negative, or zero [17]. The functions $u_{0}, v_{0}$, and $w_{0}$ are nonnegative functions which are not identically zero.

Very recently, Zhang et al. [18] found that the positive equilibrium of (3) with $r=0$ can undergo stability switch (from stable to unstable to stable, or from unstable to stable to unstable) with the change of the cannibalization rate. 
Their results indicate that large cannibalization rate can make the positive equilibrium globally stable although its stability would change with the increase of the rate. In fact, an important research subject for cannibalism models is the stabilizing/destabilizing effect of cannibalism [1, 6, 19]. For this, we need to compare the effect of cannibalism on the dynamic behavior for the ODE system, the semilinear reaction-diffusion system, and the quasi-linear cross-diffusion system.

In this paper, we first prove that stability properties of equilibria for the ODE model (3) and the semilinear reaction-diffusion model (5) is similar, but under suitable conditions on reaction coefficients, cross-diffusion-driven Turing instability in the quasi-linear model (6) occurs. It is found that the cross diffusion rate $\alpha_{13}$ is a decisive factor of destabilizing positive steady state, that is, cannibalism has no longer a stabilizing effect. Then, the uniform boundedness and the global existence of time-varying solutions for the cross-diffusion system (6) are investigated by using the energy estimates and Gagliardo-Nirenberg-type inequalities when the space dimension is one. Finally, some criteria on the global asymptotic stability of the positive equilibrium point for (6) are given by Lyapunov function. The obtained results indicate that, for any cannibalism rate $e$, under certain conditions on the other reaction coefficients, the model has no nonconstant positive steady state if the diffusion matrix is positive definite and the self-diffusion coefficients are large enough.

Moreover, we, in [14], discussed another cross-diffusion model:

$$
\left\{\begin{array}{l}
u_{t}-d_{1} \Delta u=-u+v+a u v+u w, \quad x \in \Omega, t>0, \\
v_{t}-\Delta\left(d_{2} v+\frac{d_{4} v}{\varepsilon+u^{2}}\right)=b u-g v-u v, \quad x \in \Omega, t>0, \\
w_{t}-d_{3} \Delta w=s w-r w^{2}-e u w, \quad x \in \Omega, t>0 \\
\partial_{\eta} u=\partial_{\eta} v=\partial_{\eta} w=0, \quad x \in \partial \Omega, t>0 \\
u(x, 0)=u_{0}(x) \\
v(x, 0)=v_{0}(x) \\
w(x, 0)=w_{0}(x) \\
x \in \Omega
\end{array}\right.
$$

where $d_{4}$ is a cross-diffusion coefficient and $\varepsilon$ is a positive constant (please refer to [12]). We proved that if $a, b, g, s, r, e, \varepsilon, d_{1}, d_{2}$, and $d_{3}$ are fixed such that $0<\varepsilon<\varepsilon_{0}, a g<1, r>s$ and (9) (see Section 2) hold, then there exists a positive constant $d_{4}^{*}$ such that (7) has at least one nonconstant positive steady state for $d_{4} \geq d_{4}^{*}$. This implies that the cross-diffusion rate is the decisive factor of destabilization on the constant positive steady state and therefore cannibalism is an auxiliary destabilizing force. Recently, in another paper, we detailedly describe the local structure of the nonconstant steady states and discuss the stability and instability of the steady state bifurcation.

\section{Linearization Analysis}

In this section, according to the linearization analysis and Routh-Hurwitz criterion, we focus on discussing the stability of the reaction-diffusion system (5) and cross-diffusion system (6). To this end, we first introduce the stability of the ODE system (3).

2.1. ODE Model (3). Obviously, problem (3) has trivial equilibrium $E_{0}(0,0,0)$, semitrivial equilibrium $E_{1}(0,0, s / r)$, and $E_{2}((g-b) /(a b-1),(g-b) /(a g-1), 0)$ if $g<b<1 / a$ or $g>b>1 / a$. Moreover, if $r=0, b<g+((s(1-a g)) /$ $(e+a s))$, then a positive equilibrium point is given by $E_{3}(\bar{u}, \bar{v}, \bar{w})$, where

$$
\begin{aligned}
& \bar{u}=\frac{s}{e} \\
& \bar{v}=\frac{b s}{e g+s}, \\
& \bar{w}=\frac{e(g-b)+s(1-a b)}{e g+s} .
\end{aligned}
$$

If $r \neq 0$ and

$$
\left\{\begin{array}{l}
b r+g s-g r>0, \\
s+e g+r(1-a b)>0 \\
s(1-a b)+e(g-b)>0
\end{array}\right.
$$

then a positive equilibrium point is uniquely given by $\widetilde{u}=(\tilde{u}, \widetilde{v}, \widetilde{w})$, where

$$
\begin{aligned}
& \tilde{u}=\frac{\alpha+\sqrt{\alpha^{2}+4 e \beta}}{2 e}, \\
& \widetilde{v}=\frac{b \tilde{u}}{g+\widetilde{u}}, \\
& \widetilde{w}=\frac{s-e \widetilde{u}}{r}, \\
& \alpha=a b r+s-r-e g, \\
& \beta=b r+g s-g r .
\end{aligned}
$$

In fact, the stability of these nonnegative equilibria in (3) has been obtained in [1] as follows:

(1) $E_{0}$ is unconditionally unstable.

(2) $E_{1}$ is locally asymptotically stable if $s<r$ and is unstable if $s>r$.

(3) $E_{2}$ is locally asymptotically stable if $g<b<1 / a$ and is unstable if $b<g+((s(1-a g)) /(e+a s))$. 
(4)

(a) If $a g<1$, then $E_{3}$ is locally asymptotically stable.

(b) If $a g>1, S=0$ (i.e., $e \longrightarrow \infty$ ) and $b<g$, then $E_{3}$ is locally asymptotically stable.

(c) If $a g>1, S \neq 0$ (i.e., $e \neq \infty$ ), $b<g$ and $a b<1$, then there exists $e^{*}$ such that $E_{3}$ is locally asymptotically stable when $e>e^{*}$, and is unstable when $e<e^{*}$.

Now, we give the stability of $\widetilde{u}$ with respect to model (3).

Theorem 1. The positive equilibrium $\tilde{u}$ of (3) is locally asymptotically stable if $\mathrm{ag}<1$.

Proof. The Jacobian matrix of (3) to the generic $u_{s}=\left(u_{s}\right.$, $\left.v_{s}, w_{s}\right)$ reads

$$
J\left(\mathbf{u}_{s}\right)=\left(\begin{array}{lll}
j_{11} & j_{12} & j_{13} \\
j_{21} & j_{22} & j_{23} \\
j_{31} & j_{32} & j_{33}
\end{array}\right),
$$

where

$$
\left\{\begin{array}{l}
j_{11}=-1+a v_{s}+w_{s}, \\
j_{12}=1+a u_{s}, \\
j_{13}=u_{s}, \\
j_{21}=b-v_{s}, \\
j_{22}=-g-u_{s}, \\
j_{23}=0 \\
j_{31}=-e w_{s}, \\
j_{32}=0, \\
j_{33}=s-2 r w_{s}-e u_{s} .
\end{array}\right.
$$

A direct calculation yields that the characteristic equation of $J(\widetilde{\mathbf{u}})$ is

$$
\varphi(\lambda)=|\lambda I-J|=\lambda^{3}+\alpha_{1} \lambda^{2}+\alpha_{2} \lambda+\alpha_{3}=0,
$$

with

$$
\begin{aligned}
\alpha_{1}= & \frac{1}{\widetilde{u}}\left(g \widetilde{u}+\widetilde{v}++\widetilde{u}^{2}+r \tilde{u} \widetilde{w}\right)>0, \\
\alpha_{2}= & \frac{1}{\widetilde{u}}\left((1-a g) \widetilde{u} \widetilde{v}+r \widetilde{w}(\widetilde{v}+g \widetilde{u})+(e+r) \widetilde{u}^{2} \widetilde{w}\right), \\
\alpha_{3}= & \frac{1}{\widetilde{u}}\left(e \widetilde{w} \widetilde{u}^{2}(g+\widetilde{u})+r(1-a g) \widetilde{u} \widetilde{v} \widetilde{w}\right) \\
\alpha_{1} \alpha_{2}-\alpha_{3}= & r \widetilde{w} \widetilde{u}^{2}+\left((1-a g) \widetilde{v}+2 g r \widetilde{w}+r e \widetilde{w}^{2}+r^{2} \widetilde{w}^{2}\right) \widetilde{u} \\
& +r g^{2} \widetilde{w}+e \widetilde{v} \widetilde{w}+2 r \widetilde{v} \widetilde{w}+g r^{2} \widetilde{w}^{2} \\
& +(1-a g) g \widetilde{v}+\frac{r \widetilde{w} \widetilde{v}^{2}}{\widetilde{u}^{2}}+\frac{1}{\widetilde{u}} \\
& \cdot\left((1-a g) \widetilde{v}^{2}+r^{2} \widetilde{w}^{2} \widetilde{v}+2 g r \widetilde{v} \widetilde{w}\right) .
\end{aligned}
$$

It is easy to see that $\alpha_{3}>0$ and $\alpha_{1} \alpha_{2}-\alpha_{3}>0$, thanks to $a g<1$. Therefore, by Routh-Hurwitz criterion, we know that $(\widetilde{u}, \widetilde{v}, \widetilde{w})$ is locally asymptotically stable.

Notice that the unstable equilibrium points of (3) are also unstable for (5) and (6). Therefore, for systems (5) and (6), we only discuss the stability of the equilibrium points which are stable for (3).

2.2. Weakly Coupled Reaction-Diffusion System (5). Let $0=$ $\mu_{1}<\mu_{2}<\mu_{3}<\cdots$ be the eigenvalues of the operator $-\Delta$ on $\Omega$ with the homogeneous Neumann boundary condition, and let $E\left(\mu_{i}\right)$ be the eigenspace corresponding to $\mu_{i}$ in $H^{1}(\Omega)$. Let $\mathbf{X}$ be the closure of $\left[C^{1}(\bar{\Omega})\right]^{3}$ in $\left[H^{1}(\Omega)\right]^{3}$, $\left\{\phi_{i j}: j=1,2, \ldots, \operatorname{dim} E\left(\mu_{i}\right)\right\}$ be an orthonormal basis of $E\left(\mu_{i}\right)$, and let $\mathbf{X}_{i j}=\left\{c \phi_{i j}: c \in \mathbb{R}^{3}\right\}$. Then,

$$
\begin{aligned}
\mathbf{X} & =\underset{i=1}{\oplus} X_{i}, \\
\mathbf{X}_{i} & =\underset{j=1}{\operatorname{dim} E\left(\mu_{i}\right)} \mathbf{X}_{i j} .
\end{aligned}
$$

For system (5), by the linearization analysis and some similar arguments to the proof of Theorem 2 and Theorem 3 in [12], we can obtain the following two theorems.

\section{Theorem 2}

(1) The semitrivial equilibrium $E_{1}$ of (5) is locally asymptotically stable if $s<r$

(2) The semitrivial equilibrium $E_{2}$ of (5) is locally asymptotically stable if $g<b<1 / a$

(3) The positive equilibrium $E_{3}$ of (5) is locally asymptotically stable either $a g<1$, or $a g>1, S=0$ (i.e., $e \longrightarrow \infty)$ and $b<g$

Proof. We only prove (3). The proof of (1) and (2) is similar to (3), so we omit it here. Let $\mathbf{u}=(u, v, w), \mathscr{D}=$ $\operatorname{diag}\left(d_{1}, d_{2}, d_{3}\right)$, and $\mathscr{L}=\mathscr{D} \Delta+J\left(E_{3}\right)$, where $j_{11}=-($ bel $(e g+s))<0, j_{12}=(e+a s) / e>0 . \quad j_{13}=\bar{u}>0, j_{21}=(b e g /$ $(e g+s))>0, j_{22}=-(g+\bar{u})<0, j_{23}=0, \quad j_{31}=-e \bar{w}<0$, and $j_{32}=j_{33}=0$. The linearization of (5) at $E_{3}$ is $\mathbf{u}_{t}=\mathscr{L} \mathbf{u}$. For each $i \geq 1, \mathbf{X}_{i}$ is invariant under the operator $\mathscr{L}$, and $\lambda$ is an eigenvalue of $\mathscr{L}$ if and only if it is an eigenvalue of the matrix $-\mu_{i} \mathscr{D}+J\left(E_{3}\right)$ for some $i \geq 1$, in which case there is an eigenvector in $\mathbf{X}_{i}$. Denote the characteristic polynomial of $-\mu_{i} \mathscr{D}+J\left(E_{3}\right)$ by $\varphi_{i}(\lambda)=\lambda^{3}+A_{i} \lambda^{2}+B_{i} \lambda+C_{i}$, where

$$
\begin{aligned}
A_{i}= & \mu_{i}\left(d_{1}+d_{2}+d_{3}\right)-\left(j_{11}+j_{22}\right), \\
B_{i}= & \mu_{i}^{2}\left(d_{1} d_{2}+d_{2} d_{3}+d_{1} d_{3}\right)-\mu_{i}\left(d_{1} j_{22}+d_{2} j_{11}+d_{3} j_{11}+d_{3} j_{22}\right) \\
& +j_{11} j_{22}-j_{12} j_{21}-j_{13} j_{31}, \\
C_{i}= & \mu_{i}^{3} d_{1} d_{2} d_{3}-\mu_{i}^{2}\left(d_{1} d_{3} a_{22}+d_{2} d_{3} j_{11}\right) \\
& +\mu_{i}\left(d_{3} j_{11} j_{22}-d_{3} j_{12} j_{21}-d_{2} j_{13} j_{31}\right)+j_{22} j_{13} j_{31} .
\end{aligned}
$$

A straightforward computation gives $H_{i}=A_{i} B_{i}-C_{i}=$ $I_{1} \mu_{i}^{3}+I_{2} \mu_{i}^{2}+I_{3} \mu_{i}+I_{4}$, where 


$$
\begin{aligned}
I_{1}= & d_{1}^{2}\left(d_{2}+d_{3}\right)+d_{2}^{2}\left(d_{1}+d_{3}\right)+d_{3}^{2}\left(d_{1}+d_{2}\right)+2 d_{1} d_{2} d_{3}, \\
I_{2}= & -j_{11}\left(d_{2}^{2}+d_{3}^{2}\right)-j_{22}\left(d_{1}^{2}+d_{3}^{2}\right) \\
& -2\left(j_{11}+j_{22}\right)\left(d_{1} d_{2}+d_{2} d_{3}+d_{1} d_{3}\right), \\
I_{3}= & \left(j_{11}+j_{22}\right)\left(d_{1} j_{22}+d_{2} j_{11}+d_{3} j_{11}+d_{3} j_{22}\right) \\
& +\left(d_{1}+d_{2}\right)\left(j_{11} j_{22}-j_{12} j_{21}\right)-\left(d_{1}+d_{3}\right) j_{13} j_{31}, \\
I_{4}= & -\left(j_{11}+j_{22}\right)\left(j_{11} j_{22}-j_{12} j_{21}\right)+j_{11} j_{13} j_{31} .
\end{aligned}
$$

Obviously, if $j_{11} j_{22}>j_{12} j_{21}$ (i.e., $a g<1$ ), then $H_{i}>0, A_{i}>0$, and $C_{i}>0$. It follows from the Routh-Hurwitz criterion that the three roots $\lambda_{i 1}, \lambda_{i 2}$, and $\lambda_{i 3}$ of $\varphi_{i}(\lambda)=0$ all have negative real parts. Moreover, if $S=0$, then $e=V / S \longrightarrow \infty, \bar{w} \longrightarrow 1-b / g, \quad j_{11} \longrightarrow-(b / g), j_{12} \longrightarrow$ $1, j_{13} \longrightarrow 0, j_{21} \longrightarrow b, j_{22} \longrightarrow-g$, and $j_{31} \longrightarrow-e(1-(b /$ g)). Therefore, if $b<g$, then $H_{i}>0, A_{i}>0$, and $C_{i}>0$.

We claim that, for each $i \geq 1$, there exists a positive constant $\delta$ such that

$$
\operatorname{Re}\left\{\lambda_{i 1}\right\}, \operatorname{Re}\left\{\lambda_{i, 2}\right\}, \operatorname{Re}\left\{\lambda_{i, 3}\right\} \leq-\delta, \quad i \geq 1 .
$$

Let $\quad \lambda=\mu_{i} \xi$. Then, $\quad \varphi_{i}(\lambda)=\mu_{i}^{3} \xi^{3}+A_{i} \mu_{i}^{2} \xi^{2}+B_{i} \mu_{i} \xi+$ $C_{i} \triangleq \tilde{\varphi}_{i}(\xi)$. Since $\mu_{i} \longrightarrow \infty$ as $i \longrightarrow \infty$, it follows that

$$
\begin{aligned}
\lim _{i \longrightarrow \infty}\left\{\frac{\tilde{\varphi}_{i}(\xi)}{\mu_{i}^{3}}\right\}= & \xi^{3}+\left(d_{1}+d_{2}+d_{3}\right) \xi^{2} \\
& +\left(d_{1} d_{2}+d_{2} d_{3}+d_{1} d_{3}\right) \xi+d_{1} d_{2} d_{3} \triangleq \widetilde{\varphi}(\xi) .
\end{aligned}
$$

Clearly, $\widetilde{\varphi}(\xi)=0$ has three negative roots $-d_{1},-d_{2}$, and $-d_{3}$. Let $d^{*}=\min \left\{d_{1}, d_{2}, d_{3}\right\}$. By continuity, one can see that there exists a $i_{0}$ such that the three roots $\xi_{i 1}, \xi_{i 2}$, and $\xi_{i 3}$ of $\tilde{\varphi}_{i}(\xi)=0$ satisfy $\operatorname{Re}\left\{\xi_{i 1}\right\}, \operatorname{Re}\left\{\xi_{i 2}\right\}, \operatorname{Re}\left\{\xi_{i 3}\right\} \leq-d^{*} / 2$ when $i \geq i_{0}$. Therefore, if $i \geq i_{0}$, then $\operatorname{Re}\left\{\lambda_{i 1}\right\}, \operatorname{Re}\left\{\lambda_{i 2}\right\}, \operatorname{Re}\left\{\lambda_{i 3}\right\} \leq$ $-\mu_{i} d^{*} / 2 \leq-d^{*} / 2$. Let $\widetilde{\delta}=-\max _{0 \leq i \leq i_{0}}\left\{\operatorname{Re}\left\{\lambda_{i 1}\right\}, \operatorname{Re}\left\{\lambda_{i 2}\right\}\right.$, $\left.\operatorname{Re}\left\{\lambda_{i 3}\right\}\right\}$. Then, $\tilde{\delta}>0$, and (18) holds for $\delta=\min \left\{\tilde{\delta}, d^{*} / 2\right\}$. So, the positive equilibrium point $E_{3}$ of (5) is locally asymptotically stable.
In order to obtain the global asymptotic behavior of the solutions to (5) or (6), we need the following result which can be found in [12].

Lemma 1. Let $a$ and $b$ be positive constants. Assume that $\varphi, \psi \in C^{1}[a,+\infty), \psi(t) \geq 0$, and $\varphi$ is bounded from below. If $\varphi^{\prime}(t) \leq-b \psi(t)$ and $\psi^{\prime}(t)$ is bounded from above in $[a,+\infty)$, then $\lim _{t \rightarrow \infty} \psi(t)=0$.

\section{Theorem 3}

(1) The positive equilibrium $E_{3}$ of (5) is globally asymptotically stable if $a g<1$ and $e g+a g s<e g s<e g+s$.

(2) The semitrivial equilibrium $E_{2}$ of (5) is globally asymptotically stable if $1<(1-a b)(b-g)<b / g$.

Proof. Let $(u, v, w)$ be the unique positive solution of (5). Estimates similar to Theorem 2.1 in [15] and Theorem

$A_{2}$ in [20] show

$$
\|u(\cdot, t)\|_{C^{2+\alpha}(\bar{\Omega})},\|v(\cdot, t)\|_{C^{2+\alpha}(\bar{\Omega})},\|u(\cdot, t)\|_{C^{2+\alpha}(\bar{\Omega})} \leq C, \quad \forall t \geq 1,
$$

where $\alpha \in(0,1)$ and $C$ does not depend on $t$.

(1) Define

$$
\begin{aligned}
V(u, v, w)= & \int_{\Omega}\left[\left(u-\bar{u}-\bar{u} \ln \frac{u}{\bar{u}}\right)+\lambda\left(v-\bar{v}-\bar{v} \ln \frac{v}{\bar{v}}\right)\right. \\
& \left.+\rho\left(w-\bar{w}-\bar{w} \ln \frac{w}{\bar{w}}\right)\right] \mathrm{d} x,
\end{aligned}
$$

where $\quad \lambda=(e+a s) / e g$ and $\rho=1 / e . \quad$ Obviously, $V(u, v, w)$ is nonnegative and $V(u, v, w)=0$ if and only if $u=\bar{u}, v=\bar{v}$, and $w=\bar{w}$. The time derivative of $V(u, v, w)$ for (5) satisfies that

$$
\begin{aligned}
\frac{\mathrm{d} V(u, v, w)}{\mathrm{d} t}= & -\int_{\Omega}\left(\frac{d_{1} \bar{u}}{u^{2}}|\nabla u|^{2}+\frac{\lambda d_{2} \bar{v}}{v^{2}}|\nabla v|^{2}+\frac{\rho d_{3} \bar{w}}{w^{2}}|\nabla w|^{2}\right) \mathrm{d} x \\
& -\frac{1}{s} \int_{\Omega}\left\{\frac{(e s-e-a s)}{s} \frac{v}{u}(u-\bar{u})^{2}+\frac{(e+a s)(e g+s-e g s)}{e g} \frac{u}{v}(v-\bar{v})^{2}+\left(\sqrt{\frac{(e+a s)}{s}} \frac{v}{u}(u-\bar{u})-\sqrt{s(e+a s) \frac{u}{v}}(v-\bar{v})\right)^{2}\right\} \mathrm{d} x \\
\leq & -\int_{\Omega}\left(\frac{d_{1} \bar{u}}{u^{2}}|\nabla u|^{2}+\frac{\lambda d_{2} \bar{v}}{v^{2}}|\nabla v|^{2}+\frac{\rho d_{3} \bar{w}}{w^{2}}|\nabla w|^{2}\right) \mathrm{d} x \\
& -\frac{1}{s} \int_{\Omega}\left\{\frac{(e s-e-a s)}{s} \frac{v}{u}(u-\bar{u})^{2}+\frac{(e+a s)(e g+s-e g s)}{e g} \frac{u}{v}(v-\bar{v})^{2}\right\} \mathrm{d} x .
\end{aligned}
$$


From Theorem 2, Lemma 1, and (20), we know that

$$
\begin{array}{r}
\lim _{t \rightarrow \infty} \int_{\Omega}\left(|\nabla u|^{2}+|\nabla v|^{2}+|\nabla w|^{2}\right) \mathrm{d} x=0, \\
\lim _{t \rightarrow \infty} \int_{\Omega}(u-\bar{u})^{2} \mathrm{~d} x=0, \\
\lim _{t \rightarrow \infty} \int_{\Omega}(v-\bar{v})^{2} \mathrm{~d} x=0 .
\end{array}
$$

It follows from the Poincare inequality that

$$
\begin{aligned}
& \lim _{t \rightarrow \infty} \int_{\Omega}(u-\widehat{u})^{2} \mathrm{~d} x=0, \\
& \lim _{t \rightarrow \infty} \int_{\Omega}(v-\widehat{v})^{2} \mathrm{~d} x=0, \\
& \lim _{t \rightarrow \infty} \int_{\Omega}(w-\widehat{w})^{2} \mathrm{~d} x=0,
\end{aligned}
$$

where $\widehat{f}=\int_{0}^{1} f \mathrm{~d} x$. As $|\widehat{u}-\bar{u}|^{2}=\int_{\Omega}(\widehat{u}-\bar{u})^{2} \mathrm{~d} x \leq$ $2 \int_{\Omega}(\widehat{u}-u)^{2} \mathrm{~d} x+2 \int_{\Omega}(u-\bar{u})^{2} \mathrm{~d} x$, from (24) and (25), we have

$$
\begin{aligned}
& \lim _{t \longrightarrow \infty} \widehat{u}(t)=\bar{u}, \\
& \lim _{t \rightarrow \infty} \widehat{v}(t)=\bar{v} .
\end{aligned}
$$

Therefore, there exists a sequence $\left\{t_{m}\right\}$ such that $\widehat{u}^{\prime}\left(t_{m}\right) \longrightarrow 0$ as $t_{m} \longrightarrow \infty$. Since $\left\{\widehat{w}\left(t_{m}\right)\right\}$ is bounded, so there exists a subsequence, still denoted by $\left\{\widehat{w}\left(t_{m}\right)\right\}$, such that $\widehat{w}\left(t_{m}\right) \longrightarrow \widetilde{w}$ as $t_{m} \longrightarrow \infty$.

From the first equation of (5), we have

$$
\begin{array}{r}
\widehat{u}^{\prime}\left(t_{m}\right)=\int_{\Omega}[(a v+w-1)(u-\bar{u}) \\
+(a \bar{u}+1)(v-\bar{v})+\bar{u}(w-\bar{w})]\left.\mathrm{d} x\right|_{t_{m}} .
\end{array}
$$

Let $m \longrightarrow \infty$ in the above equation, and from (25), we have

$$
\lim _{t_{m} \longrightarrow \infty} \widehat{w}(t)=\bar{w}
$$

On the contrary, (20) implies that there exists a subsequence, still denoted by $\left\{t_{m}\right\}$, and nonnegative functions $u^{*}, v^{*}, w^{*} \in C^{2}(\bar{\Omega})$ such that

$$
\begin{gathered}
\left\|u\left(\cdot, t_{m}\right)-u^{*}\right\|_{C^{2}(\bar{\Omega})} \longrightarrow 0, \\
\left\|v\left(\cdot, t_{m}\right)-v^{*}\right\|_{C^{2}(\bar{\Omega})} \longrightarrow 0, \\
\left\|w\left(\cdot, t_{m}\right)-w^{*}\right\|_{C^{2}(\bar{\Omega})} \longrightarrow 0,
\end{gathered}
$$

$m \longrightarrow \infty$.

Combining this with (25)-(28), one can obtain that $u^{*}=\bar{u}, v^{*}=\bar{v}, w^{*}=\bar{w}$, and

$$
\begin{gathered}
\left\|u\left(\cdot, t_{m}\right)-\bar{u}\right\|_{C^{2}(\bar{\Omega})} \longrightarrow 0, \\
\left\|v\left(\cdot, t_{m}\right)-\bar{v}\right\|_{C^{2}(\bar{\Omega})} \longrightarrow 0, \\
\left\|w\left(\cdot, t_{m}\right)-\bar{w}\right\|_{C^{2}(\bar{\Omega})} \longrightarrow 0,
\end{gathered}
$$

$$
m \longrightarrow \infty \text {. }
$$

The global asymptotic stability of $E_{3}$ follows from this together with Theorem 2.

(2) Define

$$
\begin{aligned}
V(u, v, w)= & \int_{\Omega}\left[\left(u-u_{2}-u_{2} \ln \frac{u}{u_{2}}\right)\right. \\
& \left.+l\left(v-v_{2}-v_{2} \ln \frac{v}{v_{2}}\right)+\frac{1}{e} w\right] \mathrm{d} x,
\end{aligned}
$$

where $l=(1-a g) /(g(1-a b))$ and $u_{2}=(g-b) /$ $(a b-1), v_{2}=(g-b) /(a g-1)$. Then,

$$
\begin{aligned}
\frac{\mathrm{d} V(u, v, w)}{\mathrm{d} t}= & -\int_{\Omega}\left(\frac{d_{1} u_{2}}{u^{2}}|\nabla u|^{2}+\frac{\lambda d_{2} v_{2}}{v^{2}}|\nabla v|^{2}\right) \mathrm{d} x-\int_{\Omega}\left\{\frac{(1-a b)(b-g)-1}{(b-g)^{2}} \frac{v}{u}\left(u-u_{2}\right)^{2}\right. \\
& +(1-a g)^{2}\left(\frac{b}{g(b-g)(1-a b)}-1\right) \frac{u}{v}\left(v-v_{2}\right)^{2}+\frac{r}{e} w^{2} \\
& \left.+\left(\frac{1}{b-g} \sqrt{\frac{v}{u}}\left(u-u_{2}\right)-(1-a g) \sqrt{\frac{u}{v}}\left(v-v_{2}\right)\right)^{2}\right\} \mathrm{d} x .
\end{aligned}
$$

From this, (2) can be proved using similar arguments as in (1).

Remark 1 . The stability of $\tilde{u}$ is demonstrated specifically in [14], that is, $\tilde{u}$ is locally asymptotically stable if $a g<1$.
2.3. Strongly Coupled Cross-Diffusion System (6). Comparing Sections 2.1 and 2.2, we find that $E_{i}, i=1,2,3$, and $\widetilde{u}$ have the same stability properties in systems (3) and (5). For the sake of convenience, we denote nonnegative equilibria $E_{i}, i=1,2,3$, and $\widetilde{u}$ by $\mathbf{u}_{s}=\left(u_{s}, v_{s}, w_{s}\right)$. Now, we 
show that the destabilization effect of cross-diffusion on $\mathbf{u}_{s}=\left(u_{s}, v_{s}, w_{s}\right)$.

Linearizing system (6) at an equilibrium $\left(u_{s}, v_{s}, w_{s}\right)$, we can obtain

$$
\begin{cases}\frac{\partial \mathbf{u}}{\partial t}=(\mathscr{D}+P) \Delta u+J u, & x \in \Omega, t>0, \\ \frac{\partial \mathbf{u}}{\partial \nu}=0, & x \in \partial \Omega, t>0, \\ \mathbf{u}(x, 0)=\mathbf{u}_{0}(x), & x \in \Omega,\end{cases}
$$

where $\mathbf{u}=(u, v, w)^{T}, \mathscr{D}=\operatorname{diag}\left(d_{1}, d_{2}, d_{3}\right)$, and $J$ is given in (11).

$$
P=\left(\begin{array}{lll}
p_{11} & p_{12} & p_{13} \\
p_{21} & p_{22} & p_{23} \\
p_{31} & p_{33} & p_{33}
\end{array}\right),
$$

with

$$
\left\{\begin{array}{l}
p_{11}=2 \alpha_{11} u_{s}+\alpha_{12} v_{s}+\alpha_{13} w_{s}, \\
p_{12}=\alpha_{12} u_{s}, \\
p_{13}=\alpha_{13} u_{s}, \\
p_{21}=\alpha_{21} v_{s}, \\
p_{22}=\alpha_{21} u_{s}+2 \alpha_{22} v_{s}+\alpha_{23} w_{s}, \\
p_{23}=\alpha_{23} v_{s}, \\
p_{31}=\alpha_{31} w_{s}, \\
p_{32}=\alpha_{32} w_{s}, \\
p_{33}=\alpha_{31} u_{s}+\alpha_{32} v_{s}+2 \alpha_{33} w_{s} .
\end{array}\right.
$$

We denote

$$
\mathscr{L}:=-\mu_{i}(\mathscr{D}+P)+J,
$$

then the corresponding characteristic polynomial of $\mathscr{L}$ is

$$
\phi=\lambda^{3}+\gamma_{1} \lambda^{2}+\gamma_{2} \lambda+\gamma_{3}
$$

where

$$
\begin{aligned}
& \gamma_{1}=(\operatorname{tr} \mathscr{D}+\operatorname{tr} P) \mu_{i}-\operatorname{tr} J, \\
& \gamma_{2}=\beta_{1} \mu_{i}^{2}+\beta_{2} \mu_{i}+\beta_{3}, \\
& \gamma_{3}=\operatorname{det}\left(\mu_{i} P-J\right)=\beta_{4} \mu_{i}^{3}+\beta_{5} \mu_{i}^{2}+\beta_{6} \mu_{i}+\beta_{7},
\end{aligned}
$$

with

$$
\begin{aligned}
\beta_{1}= & d_{1} d_{2}+d_{1} d_{3}+d_{2} d_{3}+d_{1}\left(p_{22}+p_{33}\right)+d_{2}\left(p_{11}+p_{33}\right)+d_{3}\left(p_{11}+p_{22}\right)+M_{11}+M_{22}+M_{33}, \\
\beta_{2}= & -j_{11}\left(d_{2}+d_{3}+p_{22}+p_{33}\right)-j_{22}\left(d_{1}+d_{3}+p_{11}+p_{33}\right)-j_{33}\left(d_{1}+d_{2}+p_{11}+p_{22}\right)+p_{21} j_{12}+p_{12} j_{21}+p_{31} j_{13}+p_{13} j_{31}, \\
\beta_{3}= & \bar{M}_{22}+\bar{M}_{33}+j_{22} j_{33}, \\
\beta_{4}= & \operatorname{det}(D)+\operatorname{det}(P)+d_{1}\left(d_{2} p_{33}+d_{3} p_{22}+M_{11}\right)+d_{2} M_{22}+d_{3} M_{33}+d_{2} d_{3} P_{11}, \\
\beta_{5}= & -j_{11}\left(M_{11}+d_{2} p_{33}+d_{3} p_{22}+d_{2} d_{3}\right)+j_{12}\left(M_{12}+d_{3} p_{21}\right)+j_{13}\left(d_{2} p_{31}-M_{13}\right) \\
& +j_{21}\left(d_{3} p_{12}+M_{21}\right)-j_{22}\left(M_{22}+d_{1} p_{33}+d_{3} p_{11}+d_{1} d_{3}\right) \\
& +j_{31}\left(d_{2} p_{13}-M_{31}\right)-j_{33}\left(M_{33}+d_{1} d_{2}+d_{1} p_{22}+d_{2} p_{11}\right), \\
\beta_{6}= & \left(p_{11}+d_{1}\right) j_{22} j_{33}-p_{12} j_{21} j_{33}-p_{13} j_{22} j_{31}-p_{21} j_{12} j_{33}+p_{22} j_{11} j_{33}-p_{22} j_{13} j_{31} \\
& +p_{23} j_{12} j_{31}-p_{31} j_{13} j_{22}+p_{32} j_{13} j_{21}+\left(p_{33}+d_{3}\right) \bar{M}_{33}+d_{2} \bar{M}_{22}, \\
\beta_{7}= & -j_{33} \bar{M}_{33}+j_{13} j_{22} j_{31},
\end{aligned}
$$

where $M_{i j}$ and $\bar{M}_{i j}, i, j=1,2,3$, are cofactors of matrix $P$ and $J$, respectively.

Therefore, according to the principle of the linearized stability ((21], Th 8.6), (22], Th 5.2)), the local stability of nonnegative equilibria of model (6) is given below.

Theorem 4. Let $d_{i}$ and $\alpha_{i j}, i, j=1,2,3$, be positive constants. Then, the following statements for system (6) hold.

(1) $\mathbf{u}_{s}$ is locally asymptotically stable if and only if for every $i \in \mathbb{N}$, all the eigenvalues of the linearization matrix $\mathscr{L}$ have negative real part.
(2) $\mathbf{u}_{s}$ is unstable if and only if there exists an $i \in \mathbb{N}$, such that the linearization matrix $\mathscr{L}$ has at least one eigenvalue with positive real part.

By applying the Routh-Hurwitz criterion or Corollary 2.2 in [23], we have the following stability and instability results.

\section{Corollary 1}

(1) $E_{1}$ is locally asymptotically stable if $s<r$ and $b<g m$, here $m=\min \left\{s,(r-s) / r, r s^{2} /(r-s)\right\}$.

(2) $E_{2}$ is locally asymptotically stable if $g<b<1 / a$, $(1-a b) /(1-a g)<s<e(b-g)) /(1-a b), \quad$ and 


$$
\begin{aligned}
& (a b-1)(a g-1) d_{3}+(a b-1)(g-b) \alpha_{32}+(g-b) \\
& (a g-1) \alpha_{31}>0 .
\end{aligned}
$$

(3) $E_{3}$ is locally asymptotically stable if ag $<1, e \alpha_{13}<\alpha_{31}$, and $(g+\bar{u})\left(2 \bar{w} \alpha_{33}+d_{3}\right)+\overline{u v} \alpha_{32}>s\left(\bar{u} \alpha_{21}+2 \bar{v} \alpha_{22}+\right.$ $\left.\bar{w} \alpha_{23}+d_{2}\right)$.

(4) $\tilde{u}$ is locally asymptotically stable if $s<\delta:=r(1-a b)-$ $e g+\sqrt{(a b r-r+e g)^{2}+4 e r(g-b)}, \quad b<g, a g<1$, and $(e / r) \alpha_{23} \leq \alpha_{21} \leq \alpha_{13} \leq\left(\alpha_{31} / e\right) \leq\left(\alpha_{21} \alpha_{33} / e \alpha_{23}\right)$.

(5) If $a g<1$ and $s>f$, then there exists a positive constant $\widetilde{\alpha}_{13}$ such that $\widetilde{\mathbf{u}}$ is unstable when $\alpha_{13}>\widetilde{\alpha}_{13}$.

(6) If ag $>1$, then there exists a positive constant $\tilde{d}_{3}$ such that $\widetilde{\mathbf{u}}$ is unstable when $d_{3}>\widetilde{d}_{3}$.

Proof. We only prove the case of (4) and (5), other cases can be treated similarly. With the help of the Maple application, we can evaluate that $\gamma_{i}>0, i=1,2,3$ and $\gamma_{1} \gamma_{2}-$ $\gamma_{3}>0$ due to $b<g, a g<1, \quad s<\delta:=r(1-a b)-e g+$ $\sqrt{(a b r-r+e g)^{2}+4 e r(g-b)}$ and $(e / r) \alpha_{23}<\alpha_{21}<\alpha_{13}<$ $\left(\alpha_{31} / e\right)<\left(\alpha_{21} \alpha_{33} / e \alpha_{23}\right)$. Therefore, Routh-Hurwitz criterion shows that $\widetilde{\mathbf{u}}$ is locally asymptotically stable.

On the contrary, the constant equilibrium is unstable if and only if there exists an $i \in \mathbb{N}$ such that $\gamma_{3}<0$ or $\gamma_{1} \gamma_{2}<\gamma_{3}$. Thanks to calculate $\gamma_{1} \gamma_{2}-\gamma_{3}$ is complicated, we now only discuss the case of $\gamma_{3}<0$.

In fact, $\quad \gamma_{3}=\operatorname{det}\left(\mu_{i} P-J\right)=\beta_{4} \mu_{i}^{3}+\beta_{5} \mu_{i}^{2}+\beta_{6} \mu_{i}+$ $\beta_{7}:=C\left(\alpha_{13} ; \mu_{i}\right)$, where $\beta_{4}>0$. In order to study whether cross-diffusion has unstable effect, we assume that $\widetilde{\mathbf{u}}$ is stable in the corresponding self-diffusion system, i.e., $a g<1$, which implies that $\beta_{7}=-j_{33} \widetilde{v}(1-a g)+j_{13} j_{22} j_{31}>0$. Let $\widetilde{\mu}_{1}, \widetilde{\mu}_{2}$, and $\widetilde{\mu}_{3}$ be the three roots of $C\left(\alpha_{13} ; \mu_{i}\right)$ with $\operatorname{Re}\left\{\widetilde{\mu}_{1}\right\} \leq$ $\operatorname{Re}\left\{\widetilde{\mu}_{2}\right\} \leq \operatorname{Re}\left\{\widetilde{\mu}_{3}\right\}$. Then, $\widetilde{\mu}_{1} \widetilde{\mu}_{2} \widetilde{\mu}_{3}=-\left(\beta_{4} / \beta_{1}\right)<0$, at least one of $\widetilde{\mu}_{1}, \widetilde{\mu}_{2}$, and $\widetilde{\mu}_{3}$ is real and negative, and the product of the other two is positive.

Now, we consider the following limits:

$$
\begin{aligned}
C_{1}:= & \lim _{\alpha_{13} \longrightarrow \infty} \frac{\beta_{1}}{\alpha_{13}}=2 \widetilde{w}^{3} \alpha_{23} \alpha_{3} 3+\left(2 \widetilde{u} \alpha_{21} \alpha_{33}+4 \widetilde{v} \alpha_{22} \alpha_{33}+2 d_{3} \alpha_{33}+d_{3} \alpha_{23}\right) \widetilde{w}^{2} \\
& +2 \widetilde{v} \alpha_{32}\left(\widetilde{u} \alpha_{21}+\widetilde{v} \alpha_{22}\right)+d_{2}\left(d_{3}+\widetilde{v} \alpha_{32}\right)+d_{3}\left(\widetilde{u} \alpha_{21}+2 \widetilde{v} \alpha_{22}\right), \\
C_{2}:= & \lim _{\alpha_{13} \longrightarrow \infty} \frac{\beta_{2}}{\alpha_{13}}=(r \widetilde{w}-e \widetilde{u})\left(\widetilde{u} \widetilde{w} \alpha_{21}+2 \widetilde{v} \widetilde{w} \alpha_{22}+\widetilde{w}^{2} \alpha_{23}\right) \\
& +(2 \widetilde{u} \widetilde{v}+g \widetilde{v}-b \widetilde{u}) \widetilde{w} \alpha_{32}+2 \widetilde{w}^{2}(g+u) \alpha_{33}, \\
C_{3}:= & \lim _{\alpha_{13} \longrightarrow \infty} \frac{\beta_{3}}{\alpha_{13}}=(s-2 e \widetilde{u})(g+\widetilde{u}) .
\end{aligned}
$$

Note that

$$
\lim _{\alpha_{13} \longrightarrow \infty} \frac{C\left(\alpha_{13} ; v_{i}\right)}{\alpha_{13}}=\mu_{i}\left(C_{1} \mu_{i}^{2}+C_{2} \mu_{i}+C_{3}\right),
$$

and $C_{1}>0, \quad C_{3}<0 \quad$ since $s<2 e \widetilde{u}$, i.e. $s>r(1-a b)-$ $e g+\sqrt{(a b r-r+e g)^{2}+4 e r(g-b)}$. A continuity argument yields that $\widetilde{\mu}_{1}$ is real and negative. Moreover, as $\widetilde{\mu}_{2} \widetilde{\mu}_{3}>0, \widetilde{\mu}_{2}$ and $\widetilde{\mu}_{3}$ are real and positive and

$$
\left\{\begin{array}{l}
\lim _{\alpha_{13} \longrightarrow \infty} \tilde{\mu}_{1}=\frac{-C_{2}-\sqrt{C^{2}-4 C_{1} C_{3}}}{2 C_{1}}<0, \\
\lim _{\alpha_{13} \longrightarrow \infty} \widetilde{\mu}_{2}=0, \\
\lim _{\alpha_{13} \longrightarrow \infty} \widetilde{\mu}_{3}=\frac{-C_{2}+\sqrt{C^{2}-4 C_{1} C_{3}}}{2 C_{1}}>0 .
\end{array}\right.
$$

Thus, there exists a positive constant $\widetilde{\alpha}_{13}$ such that, for all $\alpha_{13}>\widetilde{\alpha}_{13}$, the three roots $\widetilde{\mu}_{1}, \widetilde{\mu}_{2}$, and $\widetilde{\mu}_{3}$ of $C\left(\alpha_{13} ; \mu_{i}\right)$ are all real and satisfy

$$
\left\{\begin{array}{l}
-\infty<\widetilde{\mu}_{1}<0<\widetilde{\mu}_{2}<\widetilde{\mu}_{3}, \\
\alpha_{3}=C\left(\alpha_{13} ; \mu_{i}\right)<0, \quad \text { when } \mu_{i} \in\left(-\infty, \widetilde{\mu}_{1}\right) \cup\left(\widetilde{\mu}_{2}, \widetilde{\mu}_{3}\right), \\
\alpha_{3}=C\left(\alpha_{13} ; \mu_{i}\right)>0, \quad \text { when } \mu_{i} \in\left(\widetilde{\mu}_{1}, \widetilde{\mu}_{2}\right) \cup\left(\widetilde{\mu}_{3},+\infty\right) .
\end{array}\right.
$$

Therefore, the characteristic equation $\phi=0$ has at least one positive eigenvalue when $\mu_{i} \in\left(\widetilde{\mu}_{2}, \widetilde{\mu}_{3}\right)$.

Remark 2. Remark 1 and conclusion (5) of Corollary 1 indicate that if $a g<1$ and $s>f$, the large cross-diffusion rate $\alpha_{13}$ has destabilizing effect for positive equilibrium point $(\widetilde{u}, \widetilde{v}, \widetilde{w})$

This means that under suitable conditions on reaction coefficients, cross-diffusion-driven Turing instability occurs if the cross-diffusion rate is sufficiently large.

To demonstrate the results of stability and instability for $\widetilde{\mathbf{u}}$, we give the following examples.

Example 1. Assume that the spatial domain $\Omega=[0,10 \pi]$.

(1) Let 
$$
\left\{\begin{array}{l}
\alpha_{11}=\alpha_{12}=\alpha_{21}=\alpha_{22}=\alpha_{23}=\alpha_{32}=1, \\
\alpha_{13}=1.2, \\
\alpha_{31}=3, \\
\alpha_{33}=4, \\
a=0.5, \\
b=1, \\
g=1.5, \\
s=3, \\
e=2, \\
r=4, \\
d_{1}=3, \\
d_{2}=3, \\
d_{3}=2 .
\end{array}\right.
$$

Then, the equilibrium point $(\widetilde{u}, \widetilde{v}, \widetilde{w})=(0.725$, $0.326,0.388), \quad \gamma_{1}=5.809>0, \quad \gamma_{2}=10.163>0, \quad \gamma_{3}=$ $5.082>0$, and $\gamma_{1} \gamma_{2}-\gamma_{3}=53.959>0$. From Corollary 1 (4), $\widetilde{\mathbf{u}}$ is locally asymptotically stable.

(2) Let

$$
\left\{\begin{array}{l}
\alpha_{11}=\alpha_{12}=\alpha_{21}=\alpha_{22}=\alpha_{23}=\alpha_{31}=\alpha_{32}=\alpha_{33}=1, \\
d_{1}=3, \\
d_{2}=3, \\
d_{3}=2, \\
a=0.5 \\
b=1.3 \\
g=1.5 \\
s=3 \\
e=5 \\
r=4
\end{array}\right.
$$

and choose $\alpha_{13}=300$. Then, the equilibrium point $(\widetilde{u}, \widetilde{v}, \widetilde{w})=(0.453,0.302,0.184), \quad a g=0.75<1, \quad s$ $=3>f=1.195$, and $\gamma_{3}=-0.663<0$. Therefore, $\widetilde{\mathbf{u}}$ is unstable thanks to Corollary 1 (5).

(3) Let $\alpha_{i j}=0.1, i, j=1,2,3, \quad a=2.8, b=1, g=3, s=$ $3, e=3, r=4.5, d_{1}=3$, and $d_{2}=3$, and choose $d_{3}=200$. Then, the equilibrium point $(\widetilde{u}, \widetilde{v}, \widetilde{w})=$ $(0.7,0.189,0.2), a g=8.4>1$ and $\gamma_{3}=-1.643<0$. Thus, $\widetilde{\mathbf{u}}$ is unstable due to Corollary 1 (6).

\section{The Uniform Boundedness and the Global Existence of Solutions for (6)}

In this section, the uniform boundedness and the global existence of solutions for (6) are investigated when the space dimension is one. For simplicity, let $\Omega=(0,1)$, denote $|\cdot|_{k, p}=\|\cdot\|_{W_{p}^{k}(0,1)},|\cdot|_{p}=\|\cdot\|_{L_{p}(0,1)}$, and $Q_{T}=\Omega \times(0, T)$.

From the consequence of a series of important papers [24-26] by Amann, we have, if $u_{0}, v_{0}, w_{0} \in W_{p}^{1}(\Omega)$ with $p>1$, then (6) has a unique nonnegative solution $u, v, w \in C\left([0, T), W_{p}^{1}(\Omega)\right) \cap C^{\infty}\left((0, T), C^{\infty}(\Omega)\right), \quad$ where $T \leq+\infty$ is the maximal existence time for the local solution. If the solution $(u, v, w)$ satisfies the estimates

$$
\sup \left\{\|u(\cdot, t)\|_{w_{p}^{1}(\Omega)},\|v(\cdot, t)\|_{w_{p}^{1}(\Omega)},\|w(\cdot, t)\|_{w_{p}^{1}(\Omega)}: 0<t<T\right\}<\infty,
$$

then $T=+\infty$. Moreover, if $u_{0}, v_{0}, w_{0} \in W_{p}^{2}(0,1)$, then $u, v, w \in C\left([0, \infty), W_{p}^{2}(0,1)\right)$.

In order to establish the timing uniform $W_{2}^{1}$-estimate of the solution for system (6), the following corollaries to the Gagliardo-Nirenberg-type inequality play important roles.

Lemma 2. There exists a universal constant $C$ such that

$$
\begin{array}{ll}
|u|_{2} \leq C\left(\left|u_{x}\right|_{2}^{1 / 3}|u|_{1}^{2 / 3}+|u|_{1}\right), & \forall u \in W_{2}^{1}(0,1), \\
|u|_{4} \leq C\left(\left|u_{x}\right|_{2}^{1 / 2}|u|_{1}^{1 / 2}+|u|_{1}\right), & \forall u \in W_{2}^{1}(0,1), \\
|u|_{5 / 2} \leq C\left(\left|u_{x}\right|_{2}^{2 / 5}|u|_{1}^{3 / 5}+|u|_{1}\right), \quad \forall u \in W_{2}^{1}(0,1), \\
\left|u_{x}\right|_{2} \leq C\left(\left|u_{x x}\right|_{2}^{3 / 5}|u|_{1}^{2 / 5}+|u|_{1}\right), \quad \forall u \in W_{2}^{2}(0,1) .
\end{array}
$$

Theorem 5. Let $u_{0}, v_{0}, w_{0} \in W_{2}^{2}(0,1)$ and $(u, v, w)$ be the unique nonnegative solution for (6) in the maximal existence interval $[0, T)$. Assume that

(H1) $a b<1, b<g$.

$$
8 \alpha_{11} \alpha_{21} \alpha_{31}>\alpha_{21} \alpha_{13}^{2}+\alpha_{12}^{2} \alpha_{31}
$$

(H2) $8 \alpha_{12} \alpha_{22} \alpha_{32}>\alpha_{32} \alpha_{21}^{2}+\alpha_{23}^{2} \alpha_{12}$, $8 \alpha_{13} \alpha_{23} \alpha_{33}>\alpha_{23} \alpha_{31}^{2}+\alpha_{32}^{2} \alpha_{13}$.

Then, there exist $t_{0}>0$ and positive constants $M, M^{\prime}$ which depend only on $d_{i}, \alpha_{i j}(i, j=1,2,3), a, b, g, s, r, e$, such that

$$
\sup \left\{|u(\cdot, t)|_{1,2},|(v(\cdot, t))|_{1,2},|w(\cdot, t)|_{1,2}: t \in\left(t_{0}, T\right)\right\} \leq M^{\prime},
$$

$\max \left\{u(x, t), v(x, t), w(x, t):(x, t) \in[0,1] \times\left[t_{0}, T\right]\right\} \leq M$,

and $T=\infty$. Moreover, if $d_{i} \geq 1(i=1,2,3)$ and $d_{2} / d_{1}, d_{3} / d_{1} \in[\underline{d}, \bar{d}]$, where $\underline{d}$ and $\bar{d}$ are positive constants, then $M^{\prime}$ and $M$ depend on $\underline{d}$ and $\bar{d}$ but do not on $d_{1}, d_{2}$, and $d_{3}$. 
In this section, we always denote that $C$ is Sobolev embedding constant or other kind of universal constant, $A_{j}, B_{j}$, and $C_{j}$ are some positive constants which depend only on $\alpha_{i j}(i, j=1,2,3), a, b, g, s, r, e$, and $K_{j}$ are positive constants depending on $d_{i}$ and $\alpha_{i j}(i, j=1,2,3)$, $a, b, g, s, r, e$. When $d_{1}, d_{2}, d_{3} \geq 1, d_{1} / d_{2}, d_{3} / d_{2} \in[\underline{d}, \bar{d}], K_{j}$ depend on $\underline{d}$ and $\bar{d}$ but do not on $d_{1}, d_{2}$, and $d_{3}$.

Proof. By the maximum principle, one can obtain that the solutions of (6) with nonnegative initial values are always nonnegative. We will give the $W_{2}^{1}$-estimates of the solution $(u, v, w)$ for (6) next.

Firstly, we establish $L^{1}$-estimates of the solution $(u, v, w)$ for (6). Taking integrations of the first three equations in (6) over the domain $[0,1]$, respectively, and then combining the three integration equalities linearly, we have

$$
\frac{\mathrm{d}}{\mathrm{d} t} \int_{0}^{1}\left(u+\rho v+\frac{1}{e} w\right) \mathrm{d} x \leq C_{1}-l \int_{0}^{1}\left(u+\rho v+\frac{1}{e} w\right) \mathrm{d} x,
$$

where $C_{1}=\left(s^{2} / e r\right)|\Omega|, \rho=(1 / 2)(\max \{a, 1 / g\}+(1 / b))$ and $l=\min \{1-\rho b,((\rho g-1) / \rho), s\}$. It follows from $(\mathrm{H} 1)$ that there exists a positive constant $\tau_{0}$ such that

$$
\int_{0}^{1} u \mathrm{~d} x, \int_{0}^{1} v \mathrm{~d} x, \int_{0}^{1} w \mathrm{~d} x \leq M_{0}, \quad t>\tau_{0},
$$

where $M_{0}=\left(C_{1} / l\right) \max \{1,(1 / \rho), e\}$. Moreover, there exists a positive constant $M_{0}^{\prime}$ which depends on $a, b, g, s, r, e$ and the $L^{1}$-norm of $u_{0}, v_{0}$, and $w_{0}$, such that

$$
\int_{0}^{1} u \mathrm{~d} x, \int_{0}^{1} v \mathrm{~d} x, \int_{0}^{1} w \mathrm{~d} x \leq M_{0}^{\prime}, \quad t \geq 0 .
$$

Secondly, we will obtain $L^{2}$-estimates of $(u, v, w)$. We multiply the first three equations in (6) by $u, v$, and $w$, respectively, and integrate over $[0,1]$ to have

$$
\begin{aligned}
& \frac{1}{2} \frac{\mathrm{d}}{\mathrm{d} t} \int_{0}^{1} u^{2} \mathrm{~d} x \leq-d_{1} \int_{0}^{1} u_{x}^{2} \mathrm{~d} x-\int_{0}^{1} p_{11} u_{x}^{2} \mathrm{~d} x-\int_{0}^{1} p_{12} u_{x} v_{x} \mathrm{~d} x-\int_{0}^{1} p_{13} u_{x} w_{x} \mathrm{~d} x+\int_{0}^{1}\left(u v+a u^{2} v+u^{2} w\right) \mathrm{d} x \\
& \frac{1}{2} \frac{\mathrm{d}}{\mathrm{d} t} \int_{0}^{1} v^{2} \mathrm{~d} x \leq-d_{2} \int_{0}^{1} v_{x}^{2} \mathrm{~d} x-\int_{0}^{1} p_{22} v_{x}^{2} \mathrm{~d} x-\int_{0}^{1} p_{21} u_{x} v_{x} \mathrm{~d} x-\int_{0}^{1} p_{23} v_{x} w_{x} \mathrm{~d} x+b \int_{0}^{1} u v \mathrm{~d} x \\
& \frac{1}{2} \frac{\mathrm{d}}{\mathrm{d} t} \int_{0}^{1} w^{2} \mathrm{~d} x \leq-d_{3} \int_{0}^{1} w_{x}^{2} \mathrm{~d} x-\int_{0}^{1} p_{33} w_{x}^{2} \mathrm{~d} x-\int_{0}^{1} p_{31} u_{x} w_{x} \mathrm{~d} x-\int_{0}^{1} p_{32} v_{x} w_{x} \mathrm{~d} x+s \int_{0}^{1} w^{2} \mathrm{~d} x
\end{aligned}
$$

where $p_{i j}, i, j=1,2,3$, are given by (35) for $(u, v, w)$. Let

$d^{*}=\min \left\{d_{1}, d_{2}, d_{3}\right\}$. Then, we obtain that

$$
\begin{aligned}
\frac{1}{2} \frac{\mathrm{d}}{\mathrm{d} t} \int_{0}^{1}\left(u^{2}+v^{2}+w^{2}\right) \mathrm{d} x \leq & -d^{*} \int_{0}^{1}\left(u_{x}^{2}+v_{x}^{2}+w_{x}^{2}\right) \mathrm{d} x-\int_{0}^{1} q\left(u_{x}, v_{x}, w_{x}\right) \mathrm{d} x \\
& +(b+1) \int_{0}^{1} u v \mathrm{~d} x+a \int_{0}^{1} u^{2} v \mathrm{~d} x+s \int_{0}^{1} w^{2} \mathrm{~d} x
\end{aligned}
$$

where

$$
\begin{aligned}
\frac{1}{2} \frac{\mathrm{d}}{\mathrm{d} t} \int_{0}^{1}\left(u^{2}+v^{2}+w^{2}\right) \mathrm{d} x \leq & -d^{*} \int_{0}^{1}\left(u_{x}^{2}+v_{x}^{2}+w_{x}^{2}\right) \mathrm{d} x \\
& +(b+1) \int_{0}^{1} u v \mathrm{~d} x \\
& +a \int_{0}^{1} u^{2} v \mathrm{~d} x+s \int_{0}^{1} w^{2} \mathrm{~d} x .
\end{aligned}
$$$$
\begin{aligned}
q\left(u_{x}, v_{x}, w_{x}\right)= & p_{11} u_{x}^{2}+p_{22} v_{x}^{2}+p_{33} w_{x}^{2}+\left(p_{12}+p_{21}\right) u_{x} v_{x} \\
& +\left(p_{23}+p_{32}\right) v_{x} w_{x}+\left(p_{13}+p_{31}\right) u_{x} w_{x}
\end{aligned}
$$

is a positive semidefinite quadratic form of $u_{x}, v_{x}$, and $w_{x}$ if (H2) holds. So, (H2) implies that 
Now, we proceed in the following two cases:

(1) $t \geq \tau_{0}$. Notice by (48) that $|u|_{4}^{4} \leq C\left(\left|u_{x}\right|_{2}^{2}|u|_{1}^{2}+\right.$ $\left.|u|_{1}^{4}\right) \leq C M_{0}^{2}\left(\left|u_{x}\right|_{2}^{2}+M_{0}^{2}\right)$. Then, by the Young inequality,

$$
\begin{aligned}
a \int_{0}^{1} u^{2} v \mathrm{~d} x & \leq a \int_{0}^{1} \frac{u^{4}}{2 \varepsilon} \mathrm{d} x+a \int_{0}^{1} \frac{\varepsilon}{2} v^{2} \mathrm{~d} x \\
& =\frac{a^{2} C M_{0}^{2}}{2 d^{*}} \int_{0}^{1} v^{2} \mathrm{~d} x+\frac{d^{*}}{2} \int_{0}^{1} u_{x}^{2} \mathrm{~d} x+\frac{d^{*} M_{0}^{2}}{2} .
\end{aligned}
$$

Substituting (60) into (59), we have

$$
\begin{aligned}
\frac{1}{2} \frac{\mathrm{d}}{\mathrm{d} t} \int_{0}^{1}\left(u^{2}+v^{2}+w^{2}\right) \mathrm{d} x \leq & -\frac{d^{*}}{2} \int_{0}^{1}\left(u_{x}^{2}+v_{x}^{2}+w_{x}^{2}\right) \mathrm{d} x \\
& +C_{2} \int_{0}^{1}\left(u^{2}+v^{2}+w^{2}\right) \mathrm{d} x+\frac{d^{*} M_{0}^{2}}{2} .
\end{aligned}
$$

Inequality (47) implies that $|u|_{2}^{6} \leq C\left|u_{x}\right|_{2}^{2}|u|_{1}^{4}+$ $|u|_{1}^{6} \leq C M_{0}^{4}\left(\left|u_{x}\right|_{2}^{2}+M_{0}^{2}\right)$. Therefore,

$-\int_{0}^{1}\left(u_{x}^{2}+v_{x}^{2}+w_{x}^{2}\right) \mathrm{d} x \leq 3 M_{0}^{2}-\frac{1}{9 C M_{0}^{2}}\left[\int_{0}^{1}\left(u^{2}+v^{2}+w^{2}\right) \mathrm{d} x\right]^{3}$,

which together with (59) infers that

$$
\begin{aligned}
\frac{1}{2} \frac{\mathrm{d}}{\mathrm{d} t} \int_{0}^{1}\left(u^{2}+v^{2}+w^{2}\right) \mathrm{d} x \leq & -C_{3} d^{*}\left[\int_{0}^{1}\left(u^{2}+v^{2}+w^{2}\right) \mathrm{d} x\right]^{3} \\
& +C_{2} \int_{0}^{1}\left(u^{2}+v^{2}+w^{2}\right) \mathrm{d} x+2 d^{*} M_{0}^{2} .
\end{aligned}
$$

This means that there exist positive constants $\tau_{1} \geq \tau_{0}$ and $M_{1}$ depending on $a, b, g, s, r, e, d_{i}(i=1,2,3)$ such that

$$
\int_{0}^{1} u^{2} \mathrm{~d} x, \int_{0}^{1} v^{2} \mathrm{~d} x, \int_{0}^{1} w^{2} \mathrm{~d} x \leq M_{1}, \quad t \geq \tau_{1} .
$$

When $d^{*} \geq 1, M_{1}$ is independent of $d^{*}$ since the zero point of the right-hand side in (63) can be estimated by positive constants independent of $d^{*}$.

(2) $t \geq 0$. Replacing $M_{0}$ with $M_{0}^{\prime}$ and repeating estimates (60)-(63), one can obtain a new inequality which is similar to (64). The coefficients of this new inequality depend not only on $d_{i}(i=1,2,3), a, b, g, s, r, e$ but also on initial functions $u_{0}, v_{0}, w_{0}$. Then, there exists a positive constant $M_{1}^{\prime}$ depending on $a, b, g, s, r, e$, $d_{i}(i=1,2,3)$ and the $L^{2}$-norm of $u_{0}, v_{0}$, and $w_{0}$ such that

$$
\int_{0}^{1} u^{2} \mathrm{~d} x, \int_{0}^{1} v^{2} \mathrm{~d} x, \int_{0}^{1} w^{2} \mathrm{~d} x \leq M_{1}^{\prime}, \quad t \geq 0 .
$$

Finally, $L^{2}$-estimates of $u_{x}, v_{x}$, and $w_{x}$ will be obtained. We introduce the following scaling:

$$
\begin{aligned}
& \widetilde{u}=\frac{u}{d_{2}}, \\
& \widetilde{v}=\frac{v}{d_{2}}, \\
& \widetilde{w}=\frac{w}{d_{2}}, \\
& \widetilde{t}=d_{1} t .
\end{aligned}
$$

Denoting $\xi=d_{2} / d_{1}$ and $\eta=d_{3} / d_{1}$ and using $u, v, w$, and $t$ instead of $\widetilde{u}, \widetilde{v}, \widetilde{w}$, and $\widetilde{t}$, respectively, then system (6) reduces to

$$
\left\{\begin{array}{l}
u_{t}=P_{x x}+f(u, v, w), \quad 0<x<1, t>0 \\
v_{t}=Q_{x x}+k(u, v, w), \quad 0<x<1, t>0 \\
w_{t}=R_{x x}+h(u, v, w), \quad 0<x<1, t>0 \\
u_{x}(x, t)=v_{x}(x, t)=w_{x}(x, t)=0, \quad x=0,1, t>0 \\
u(x, 0)=u_{0}(x) \\
v(x, 0)=v_{0}(x) \\
w(x, 0)=w_{0}(x) \\
0<x<1
\end{array}\right.
$$

where $P=u+\alpha_{11} \xi u^{2}+\alpha_{12} \xi u v+\alpha_{13} \xi u w, Q=\xi v+\alpha_{21} \xi u v+$ $\alpha_{22} \xi v^{2}+\alpha_{23} \xi v w, R=\eta w+\alpha_{31} \xi u w+\alpha_{32} \xi v w+\alpha_{33} \xi w^{2}$, and $f(u, v, w)=-d_{1}^{-1} u+d_{1}^{-1} v+a \xi u v+\xi u w, k(u, v, w)=b d_{1}^{-1}$ $u-g d_{1}^{-1} v-\xi u v, h(u, v, w)=w\left(s d_{1}^{-1}-r \xi w-e \xi u\right)$.

We still divide the subsequent discuss into two cases:

(1) $t \geq \tau_{1}^{*}\left(=d_{1} \tau_{1}\right)$ (namely, $t \geq \tau_{1}$ in the original scale). It is clear that

$$
\begin{array}{r}
\int_{0}^{1} u \mathrm{~d} x, \int_{0}^{1} v \mathrm{~d} x, \int_{0}^{1} w \mathrm{~d} x \leq M_{0} d_{2}^{-1}, \\
\int_{0}^{1} u^{2} \mathrm{~d} x, \int_{0}^{1} v^{2} \mathrm{~d} x, \int_{0}^{1} w^{2} \mathrm{~d} x \leq M_{1} d_{2}^{-2}, \\
|P|_{1},|Q|_{1},|R|_{1} \leq D K_{1} d_{2}^{-1} .
\end{array}
$$

Multiplying the first three equations in (67) by $P_{t}, Q_{t}$, and $R_{t}$ and integrating them over the domain $[0,1]$, respectively, then adding up the three integration equalities, we have

$$
\begin{aligned}
\frac{1}{2} \bar{y}^{\prime}(t)= & -\int_{0}^{1} u_{t}^{2} \mathrm{~d} x-\xi \int_{0}^{1} v_{t}^{2} \mathrm{~d} x \\
& -\eta \int_{0}^{1} w_{t}^{2} \mathrm{~d} x-\xi \int_{0}^{1} q\left(u_{t}, v_{t}, w_{t}\right) \mathrm{d} x \\
& +\int_{0}^{1}\left[\left(1+p_{11} \xi\right) u_{t} f+p_{12} \xi v_{t} f+p_{13} \xi w_{t} f\right] \mathrm{d} x \\
& +\xi \int_{0}^{1}\left[p_{21} u_{t} k+\left(1+p_{22}\right) v_{t} k+p_{23} w_{t} k\right] \mathrm{d} x \\
& +\int_{0}^{1}\left[p_{31} \xi u_{t} h+p_{32} \xi v_{t} h+\left(\eta+p_{33} \xi\right) w_{t} h\right] \mathrm{d} x,
\end{aligned}
$$


where $\bar{y}=\int_{0}^{1}\left(P_{x}^{2}+Q_{x}^{2}+R_{x}^{2}\right) \mathrm{d} x, q$ is given by (58). It is not hard to verify by (H2) that there exists a positive constant $C_{4}$ depending only on $\alpha_{i j}(i, j=$ $1,2,3)$ such that

$$
q\left(u_{t}, v_{t}, w_{t}\right) \geq C_{4}(u+v+w)\left(u_{t}^{2}+v_{t}^{2}+w_{t}^{2}\right) .
$$

Therefore,

$$
\begin{aligned}
\frac{1}{2} \bar{y}^{\prime}(t) \leq & -\int_{0}^{1} u_{t}^{2} \mathrm{~d} x-\xi \int_{0}^{1} v_{t}^{2} \mathrm{~d} x-\eta \int_{0}^{1} w_{t}^{2} \mathrm{~d} x-C_{4} \xi \int_{0}^{1}(u+v+w)\left(u_{t}^{2}+v_{t}^{2}+w_{t}^{2}\right) \mathrm{d} x \\
& +\int_{0}^{1}\left(1+p_{11} \xi\right) u_{t} f \mathrm{~d} x+\int_{0}^{1} \xi\left(1+p_{22}\right) v_{t} k \mathrm{~d} x+\int_{0}^{1}\left(\eta+p_{33} \xi\right) w_{t} h \mathrm{~d} x \\
& +\int_{0}^{1} p_{12} \xi v_{t} f \mathrm{~d} x+\int_{0}^{1} p_{13} \xi w_{t} f \mathrm{~d} x+\int_{0}^{1} p_{21} \xi u_{t} k \mathrm{~d} x \\
& +\int_{0}^{1} p_{23} \xi w_{t} k \mathrm{~d} x+\int_{0}^{1} p_{31} \xi u_{t} h \mathrm{~d} x+\int_{0}^{1} p_{32} \xi v_{t} h \mathrm{~d} x
\end{aligned}
$$

By the Hölder inequality, one can obtain the fol-

lowing estimates:

$$
\begin{aligned}
& \int_{0}^{1} u^{4} \mathrm{~d} x \leq M_{1}^{1 / 3} d_{1}^{-(2 / 3)}\left(\int_{0}^{1} u^{5} \mathrm{~d} x\right)^{2 / 3}, \\
& \int_{0}^{1} u^{2} v^{2} \mathrm{~d} x \leq M_{1}^{1 / 3} d_{1}^{-(2 / 3)}\left(\int_{0}^{1} u^{5} \mathrm{~d} x\right)^{1 / 3}\left(\int_{0}^{1} v^{5} \mathrm{~d} x\right)^{1 / 3}, \\
& \int_{0}^{1} u^{3} \mathrm{~d} x \leq M_{1}^{2 / 3} d_{1}^{-(4 / 3)}\left(\int_{0}^{1} u^{5} \mathrm{~d} x\right)^{1 / 3}, \\
& \int_{0}^{1} u v^{2} \mathrm{~d} x \leq M_{1}^{2 / 3} d_{1}^{-(4 / 3)}\left(\int_{0}^{1} v^{5} \mathrm{~d} x\right)^{1 / 3}, \\
& \int_{0}^{1} u^{3} w \mathrm{~d} x \leq M_{1}^{1 / 3} d_{2}^{-(2 / 3)}\left(\int_{0}^{1} u^{5} \mathrm{~d} x\right)^{1 / 2}\left(\int_{0}^{1} w^{5} \mathrm{~d} x\right)^{1 / 6}, \\
& \int_{0}^{1} u v w \mathrm{~d} x \leq M_{1}^{2 / 3} d_{2}^{-(4 / 3)}\left(\int_{0}^{1} u^{5} \mathrm{~d} x\right)^{1 / 6}\left(\int_{0}^{1} v^{5} \mathrm{~d} x\right)^{1 / 6}, \\
& \int_{0}^{1} u^{2} v w \mathrm{~d} x \leq M_{1}^{1 / 3} d_{2}^{-(2 / 3)}\left(\int_{0}^{1} u^{5} \mathrm{~d} x\right)^{1 / 3}\left(\int_{0}^{1} v^{5} \mathrm{~d} x\right)^{1 / 6}\left(\int_{0}^{1} w^{5} \mathrm{~d} x\right)^{1 / 6} .
\end{aligned}
$$

Applying the Young inequality, the Hölder inequality, the Gagliardo-Nirenberg inequality, and estimate (68), one can obtain the following estimates for the terms on the right-hand side of (71): 
Complexity

13

$$
\begin{aligned}
-\int_{0}^{1} u_{t}^{2} \mathrm{~d} x \leq & -\frac{1}{2} \int_{0}^{1} P_{x x}^{2} \mathrm{~d} x+\int_{0}^{1} f^{2} \mathrm{~d} x, \\
-\xi \int_{0}^{1} v_{t}^{2} \mathrm{~d} x \leq & -\frac{\xi}{2} \int_{0}^{1} Q_{x x}^{2} \mathrm{~d} x+\xi \int_{0}^{1} k^{2} \mathrm{~d} x, \\
-\eta \int_{0}^{1} w_{t}^{2} \mathrm{~d} x \leq & -\frac{\eta}{2} \int_{0}^{1} R_{x x}^{2} \mathrm{~d} x+\eta \int_{0}^{1} h^{2} \mathrm{~d} x \\
\int_{0}^{1} f^{2} \mathrm{~d} x \leq & 2 M_{1} d_{1}^{-2} d_{2}^{-2}+a^{2} \xi^{2} M_{1}^{1 / 3} d_{2}^{-(2 / 3)}\left(\int_{0}^{1} u^{5} \mathrm{~d} x\right)^{1 / 3}\left(\int_{0}^{1} v^{5} \mathrm{~d} x\right)^{1 / 3} \\
& +\xi^{2} M_{1}^{1 / 3} d_{2}^{-(2 / 3)}\left(\int_{0}^{1} u^{5} \mathrm{~d} x\right)^{1 / 3}\left(\int_{0}^{1} w^{5} \mathrm{~d} x\right)^{1 / 3}+2 a \xi d_{1}^{-1} d_{2}^{-(4 / 3)} M_{1}^{2 / 3}\left(\int_{0}^{1} v^{5} \mathrm{~d} x\right)^{1 / 3} \\
& +2 \xi d_{1}^{-1} d_{2}^{-(2 / 3)} M_{1}^{2 / 3}\left(\int_{0}^{1} u^{5} \mathrm{~d} x\right)^{1 / 6}\left(\int_{0}^{1} v^{5} \mathrm{~d} x\right)^{1 / 6} \\
& +2 a \xi^{2} d_{2}^{-(2 / 3)} M_{1}^{1 / 3}\left(\int_{0}^{1} u^{5} \mathrm{~d} x\right)^{1 / 3}\left(\int_{0}^{1} v^{5} \mathrm{~d} x\right)^{1 / 6}\left(\int_{0}^{1} w^{5} \mathrm{~d} x\right)^{1 / 6} \\
& +\eta r^{2} \xi^{2} d_{2}^{-(2 / 3)} M_{1}^{1 / 3}\left(\int_{0}^{1} u^{5} \mathrm{~d} x\right)^{2 / 3}+\eta d r \xi^{2} d_{2}^{-(2 / 3)} M_{1}^{1 / 3}\left(\int_{0}^{1} u^{5} \mathrm{~d} x\right)^{1 / 6}\left(\int_{0}^{1} w^{5} \mathrm{~d} x\right)^{1 / 2} \\
& +\xi^{2} g d_{1}^{-1} d_{2}^{-(4 / 3)} M_{1}^{2 / 3}\left(\int_{0}^{1} v^{5} \mathrm{~d} x\right)^{1 / 3}, \\
\xi \int_{0}^{1} k^{2} \mathrm{~d} x \leq & \left.\xi d_{1}^{-2} d_{2}^{-2} M_{1}\left(b^{2} \mathrm{~d} x \leq g^{2}\right)+\xi^{3} d_{2}^{-(2 / 3)} d_{1}^{-2} d_{2}^{-2} M_{1}+\eta d_{0}^{2} \xi^{2} d_{2}^{-(2 / 3)} M_{1}^{1 / 3}\left(\int_{0}^{1} u^{5} \mathrm{~d} x\right)^{1 / 3} \eta r^{2} \xi^{2} d_{2}^{-(2 / 3)} M_{1}^{1 / 3} \mathrm{~d} x\right)^{1 / 3}
\end{aligned}
$$

Thus,

$$
\begin{aligned}
-\int_{0}^{1} u_{t}^{2} \mathrm{~d} x-\xi \int_{0}^{1} v_{t}^{2} \mathrm{~d} x-\eta \int_{0}^{1} w_{t}^{2} \mathrm{~d} x \leq & -\frac{1}{2} \int_{0}^{1} P_{x x}^{2} \mathrm{~d} x-\frac{\xi}{2} \int_{0}^{1} Q_{x x}^{2} \mathrm{~d} x-\frac{\eta}{2} \int_{0}^{1} R_{x x}^{2} \mathrm{~d} x+C_{5}(1+\xi+\eta) d_{1}^{-2} d_{2}^{-2} M_{1} \\
& +C_{6} d_{2}^{-(4 / 3)} M_{1}^{2 / 3} \xi(1+\eta)\left(\int_{0}^{1}\left(u^{5}+v^{5}\right) \mathrm{d} x\right)^{1 / 3} \\
& +C_{7} d_{2}^{-(2 / 3)} M_{1}^{1 / 3} \xi^{2}(1+\eta)\left(\int_{0}^{1}\left(u^{5}+v^{5}+w^{5}\right) \mathrm{d} x\right)^{2 / 3}
\end{aligned}
$$




$$
\begin{aligned}
& \int_{0}^{1} u_{t} f \mathrm{~d} x \leq d_{1}^{-1}\left|\int_{0}^{1} u u_{t} \mathrm{~d} x\right|+d_{1}^{-1}\left|\int_{0}^{1} u_{t} v \mathrm{~d} x\right|+a \xi\left|\int_{0}^{1} u u_{t} v \mathrm{~d} x\right|+\xi\left|\int_{0}^{1} u u_{t} w \mathrm{~d} x\right| \\
& \leq d_{1}^{-1}\left(\frac{1}{2 \epsilon} \int_{0}^{1} u \mathrm{~d} x+\frac{\epsilon}{2} \int_{0}^{1} u u_{t}^{2} \mathrm{~d} x\right)+d_{1}^{-1}\left(\frac{1}{2 \epsilon} \int_{0}^{1} v \mathrm{~d} x+\frac{\epsilon}{2} \int_{0}^{1} v u_{t}^{2} \mathrm{~d} x\right) \\
& +a \xi\left(\frac{1}{2 \epsilon} \int_{0}^{1} u v^{2} \mathrm{~d} x+\frac{\epsilon}{2} \int_{0}^{1} u u_{t}^{2} \mathrm{~d} x\right)+\xi\left(\frac{1}{2 \epsilon} \int_{0}^{1} u w^{2} \mathrm{~d} x+\frac{\epsilon}{2} \int_{0}^{1} u u_{t}^{2} \mathrm{~d} x\right) \\
& \leq \frac{d_{1}^{-1} d_{2}^{-1}}{\epsilon} M_{0}+\frac{a}{2 \epsilon} M_{1}^{2 / 3} d_{2}^{-(4 / 3)} \xi\left(\int_{0}^{1} v^{5} \mathrm{~d} x\right)^{1 / 3}+\frac{\xi}{2 \epsilon} M_{1}^{2 / 3} d_{2}^{-(4 / 3)}\left(\int_{0}^{1} w^{5} \mathrm{~d} x\right)^{1 / 3} \\
& +\frac{\epsilon d_{1}^{-1}}{2} \int_{0}^{1} v u_{t}^{2} \mathrm{~d} x+\frac{\epsilon}{2}\left(d_{1}^{-1}+a \xi+\xi\right) \int_{0}^{1} u u_{t}^{2} \mathrm{~d} x \\
& 2 \alpha_{11} \xi \int_{0}^{1} u u_{t} f \mathrm{~d} x \leq \frac{\alpha_{11}}{\epsilon} M_{1}^{2 / 3} d_{1}^{-1} d_{2}^{2 / 3}\left[\left(\int_{0}^{1} u^{5} \mathrm{~d} x\right)^{1 / 3}+\left(\int_{0}^{1} v^{5} \mathrm{~d} x\right)^{1 / 3}\right]+\frac{3 \alpha_{11}(a+1)}{5 \epsilon} \xi^{2} \int_{0}^{1} u^{5} \mathrm{~d} x \\
& +\frac{2 \alpha_{11} a}{5 \epsilon} \xi^{2} \int_{0}^{1} v^{5} d x+\frac{2 \alpha_{11}}{5 \epsilon} \xi^{2} \int_{0}^{1} w^{5} \mathrm{~d} x+\alpha_{11} \xi \epsilon\left(2 d_{1}^{-1}+a \xi+\xi\right) \int_{0}^{1} u u_{t}^{2} \mathrm{~d} x, \\
& 2 \alpha_{12} \xi \int_{0}^{1} v u_{t} f \mathrm{~d} x \leq \frac{\alpha_{12}}{\epsilon} M_{1}^{2 / 3} d_{1}^{-1} d_{2}^{-(4 / 3)}\left(\int_{0}^{1} v^{5} \mathrm{~d} x\right)^{1 / 3}+\frac{\alpha_{12}(a+1)}{5 \epsilon} \xi^{2} \int_{0}^{1} u^{5} \mathrm{~d} x+\frac{2 \alpha_{12}}{5 \epsilon} \xi^{2} \int_{0}^{1} w^{5} \mathrm{~d} x+\frac{\alpha_{12}(3 a+4)}{10 \epsilon} \xi^{2} \int_{0}^{1} v^{5} \mathrm{~d} x, \\
& \alpha_{13} \xi \int_{0}^{1} w u_{t} f \mathrm{~d} x \leq \frac{\alpha_{13}}{\epsilon} M_{1}^{2 / 3} d_{1}^{-1} d_{2}^{-(4 / 3)}\left(\int_{0}^{1} w^{5} \mathrm{~d} x\right)^{1 / 3}+\frac{\alpha_{13}(a+(1 / 2))}{5 \epsilon} \xi^{2} \int_{0}^{1} u^{5} \mathrm{~d} x+\frac{2 a \alpha_{13}}{5 \epsilon} \xi^{2} \int_{0}^{1} v^{5} \mathrm{~d} x \\
& +\frac{2 \alpha_{13}(a+1)}{5 \epsilon} \xi^{2} \int_{0}^{1} w^{5} \mathrm{~d} x+\frac{\alpha_{13} d_{1}^{-1}}{2} \epsilon \xi \int_{0}^{1} v u_{t}^{2} \mathrm{~d} x+\frac{\epsilon}{2}\left(\alpha_{13} \xi d_{1}^{-1}+\alpha_{13} \xi^{2}+1\right) \int_{0}^{1} u u_{t}^{2} \mathrm{~d} x, \\
& \xi \int_{0}^{1} v_{t} k \mathrm{~d} x \leq \frac{b+g}{2 \epsilon} M_{0} d_{1}^{-1} d_{2}^{-1} \xi+\frac{\xi^{2}}{2 \epsilon} M_{1}^{2 / 3} d_{2}^{-(4 / 3)}\left(\int_{0}^{1} u^{5} \mathrm{~d} x\right)^{1 / 3}+\frac{b d_{1}^{-1}}{2} \epsilon \xi \int_{0}^{1} u v_{t}^{2} \mathrm{~d} x+\frac{\epsilon}{2} \xi\left(g d_{1}^{-1}+\xi\right) \int_{0}^{1} v v_{t}^{2} \mathrm{~d} x \\
& \alpha_{21} \xi \int_{0}^{1} u v_{t} k \mathrm{~d} x \leq \frac{\alpha_{21}(b+g)}{2 \epsilon} M_{1}^{2 / 3} d_{1}^{-1} d_{2}^{-(4 / 3)}\left(\int_{0}^{1} u^{5} \mathrm{~d} x\right)^{1 / 3}+\frac{2 \alpha_{21}}{5 \epsilon} \xi^{2} \int_{0}^{1} u^{5} \mathrm{~d} x \\
& +\frac{\alpha_{21}}{10 \epsilon} \xi^{2} \int_{0}^{1} v^{5} \mathrm{~d} x+\frac{\alpha_{21} b d_{1}^{-1}}{2} \epsilon \xi \int_{0}^{1} u v_{t}^{2} \mathrm{~d} x+\frac{\alpha_{21} \epsilon \xi}{2}\left(g d_{1}^{-1}+\xi\right) \int_{0}^{1} v v_{t}^{2} \mathrm{~d} x, \\
& 2 \alpha_{22} \xi \int_{0}^{1} v v_{t} k d x \leq \frac{\alpha_{22}(b+g)}{\epsilon} M_{1}^{2 / 3} d_{1}^{-1} d_{2}^{-(4 / 3)}\left(\int_{0}^{1} u^{5} \mathrm{~d} x\right)^{1 / 3}+\frac{\alpha_{22}}{5 \epsilon} \xi^{2} \int_{0}^{1} u^{5} \mathrm{~d} x \\
& +\frac{4 \alpha_{22}}{5 \epsilon} \xi^{2} \int_{0}^{1} v^{5} \mathrm{~d} x+\alpha_{22}(b+g) d_{1}^{-1} \epsilon \xi \int_{0}^{1} v v_{t}^{2} \mathrm{~d} x+\alpha_{22} \epsilon \xi^{2} \int_{0}^{1} u v_{t}^{2} \mathrm{~d} x \\
& \alpha_{23} \xi \int_{0}^{1} w v_{t} k \mathrm{~d} x \leq \frac{\alpha_{23}(b+g)}{2 \epsilon} M_{1}^{2 / 3} d_{1}^{-1} d_{2}^{-(4 / 3)}\left(\int_{0}^{1} w^{5} \mathrm{~d} x\right)^{-(4 / 3)}+\frac{\alpha_{23} \epsilon \xi}{2}\left(b d_{1}^{-1}+\xi\right) \int_{0}^{1} u v_{t}^{2} \mathrm{~d} x \\
& +\frac{\alpha_{23}}{5 \epsilon} \xi^{2}\left(\int_{0}^{1} u^{5} \mathrm{~d} x+2 \int_{0}^{1} v^{5} \mathrm{~d} x+2 \int_{0}^{1} w^{5} \mathrm{~d} x\right)+\frac{\alpha_{23} g d_{1}^{-1}}{2} \epsilon \xi \int_{0}^{1} v v_{t}^{2} \mathrm{~d} x \\
& \eta \int_{0}^{1} w_{t} h \mathrm{~d} x \leq \frac{s}{\epsilon} d_{1}^{-1} d_{2}^{-1} M_{0}+\frac{r}{2 \epsilon} M_{1}^{2 / 3} d_{2}^{-(4 / 3)} \eta \xi\left(\int_{0}^{1} w^{5} \mathrm{~d} x\right)^{1 / 3} \\
& +\frac{e}{2 \epsilon} M_{1}^{2 / 3} d_{2}^{-(4 / 3)} \eta \xi\left(\int_{0}^{1} w^{5} \mathrm{~d} x\right)^{1 / 3} \frac{\eta \epsilon}{2}\left(s d_{1}^{-1}+r \xi+e \xi\right) \int_{0}^{1} w w_{t}^{2} \mathrm{~d} x, \\
& \alpha_{31} \xi \int_{0}^{1} u w_{t} h \mathrm{~d} x \leq \frac{\alpha_{31} s}{2 \epsilon} M_{1}^{2 / 3} d_{1}^{-1} d_{2}^{-(4 / 3)} \xi\left(\int_{0}^{1} u^{5} \mathrm{~d} x\right)^{1 / 3} \\
& +\frac{\alpha_{31}(r+2 e)}{5 \epsilon} \xi^{2}\left(\int_{0}^{1} u^{5} \mathrm{~d} x\right)^{1 / 3}+\frac{\alpha_{31}(3 r+e)}{10 €} \xi^{2}\left(\int_{0}^{1} w^{5} \mathrm{~d} x\right)^{1 / 3} \\
& +\frac{\alpha_{31} \xi \epsilon}{2}\left(s d_{1}^{-1}+r \xi+e \xi\right) \int_{0}^{1} w w_{t}^{2} \mathrm{~d} x
\end{aligned}
$$


Complexity

15

$$
\begin{aligned}
& \alpha_{32} \xi \int_{0}^{1} v w_{t} h \mathrm{~d} x \leq \frac{\alpha_{32} s}{2 \epsilon} M_{1}^{2 / 3} d_{1}^{-1} d_{2}^{-(4 / 3)} \xi\left(\int_{0}^{1} v^{5} \mathrm{~d} x\right)^{1 / 3}+\frac{2 \alpha_{32} e}{5 \epsilon} \xi^{2} \int_{0}^{1} u^{5} \mathrm{~d} x+\frac{\alpha_{32}(r+2 e)}{5 \epsilon} \xi^{2} \int_{0}^{1} v^{5} \mathrm{~d} x \\
& +\frac{\alpha_{32}(e+(3 r / 2))}{5 \epsilon} \xi^{2} \int_{0}^{1} w^{5} \mathrm{~d} x+\frac{\alpha_{32} \epsilon \xi}{2}\left(s d_{1}^{-1}+r \xi+e \xi\right) \int_{0}^{1} w w_{t}^{2} \mathrm{~d} x \\
& 2 \alpha_{33} \xi \int_{0}^{1} w w_{t} h \mathrm{~d} x \leq \frac{\alpha_{33} s}{\epsilon} M_{1}^{2 / 3} d_{1}^{-1} d_{2}^{-(4 / 3)} \xi\left(\int_{0}^{1} w^{5} \mathrm{~d} x\right)^{1 / 3}+\frac{2 \alpha_{33} e^{2}}{5 \epsilon} \xi^{2} \\
& +\frac{\alpha_{33}(5 r+3 e)}{5 \epsilon} \xi^{2} \int_{0}^{1} w^{5} \mathrm{~d} x+\alpha_{33} \epsilon \xi\left(s d_{1}^{-1}+r \xi+e \xi\right) \int_{0}^{1} w w_{t}^{2} \mathrm{~d} x, \\
& \alpha_{12} \xi \int_{0}^{1} u v_{t} f \mathrm{~d} x \leq \frac{\alpha_{12}}{\epsilon} M_{1}^{2 / 3} d_{1}^{-1} d_{2}^{-(4 / 3)}\left(\int_{0}^{1} u^{5} \mathrm{~d} x\right)^{1 / 3}+\frac{\alpha_{12}}{5 \epsilon}\left(2 a+\frac{3}{2}\right) \xi^{2} \int_{0}^{1} u^{5} \mathrm{~d} x \\
& +\frac{a \alpha_{12}}{10 \epsilon} \xi^{2} \int_{0}^{1} v^{5} \mathrm{~d} x+\frac{\alpha_{12}}{5 \epsilon} \xi^{2} \int_{0}^{1} w^{5} \mathrm{~d} x+\frac{\alpha_{12} \epsilon \xi}{2}\left(d_{1}^{-1}+\xi\right) \int_{0}^{1} u v_{t}^{2} \mathrm{~d} x+\frac{\alpha_{12} \epsilon \xi}{2}\left(d_{1}^{-1}+a \xi\right) \int_{0}^{1} v v_{t}^{2} \mathrm{~d} x, \\
& \alpha_{12} \xi \int_{0}^{1} u w_{t} f \mathrm{~d} x \leq \frac{\alpha_{12} \xi}{2 \epsilon} M_{1}^{2 / 3} d_{1}^{-1} d_{2}^{-(4 / 3)}\left[\left(\int_{0}^{1} u^{5} \mathrm{~d} x\right)^{1 / 3}+\left(\int_{0}^{1} v^{5} \mathrm{~d} x\right)^{1 / 3}\right] \\
& +\frac{3 \alpha_{12}(a+1)}{10 \epsilon} \xi^{2} \int_{0}^{1} u^{5} \mathrm{~d} x+\frac{a \alpha_{12}}{5 \epsilon} \xi^{2} \int_{0}^{1} v^{5} \mathrm{~d} x+\frac{\alpha_{12}}{5 \epsilon} \xi^{2} \int_{0}^{1} w^{5} \mathrm{~d} x+\alpha_{12} \xi \epsilon\left(d_{1}^{-1}+\frac{a \xi}{2}+\frac{\xi}{2}\right) \int_{0}^{1} u w_{t}^{2} \mathrm{~d} x \\
& \alpha_{21} \xi \int_{0}^{1} v u_{t} k \mathrm{~d} x \leq \frac{\alpha_{21} \xi}{2 \epsilon} M_{1}^{2 / 3} d_{1}^{-1} d_{2}^{-(4 / 3)}\left[b\left(\int_{0}^{1} u^{5} \mathrm{~d} x\right)^{1 / 3}+g\left(\int_{0}^{1} v^{5} \mathrm{~d} x\right)^{1 / 3}\right] \\
& +\frac{\alpha_{21}}{5 \epsilon} \xi^{2} \int_{0}^{1} u^{5} \mathrm{~d} x+\frac{3 \alpha_{21}}{10 \epsilon} \xi^{2} \int_{0}^{1} v^{5} \mathrm{~d} x+\frac{\alpha_{21} \epsilon \xi}{2}\left(b d_{1}^{-1}+g d_{1}^{-1}+\xi\right) \int_{0}^{1} v u_{t}^{2} \mathrm{~d} x, \\
& \alpha_{23} \xi \int_{0}^{1} v w_{t} k \mathrm{~d} x \leq \frac{\alpha_{23} \xi}{2 \epsilon} M_{1}^{2 / 3} d_{1}^{-1} d_{2}^{-(4 / 3)}\left[b\left(\int_{0}^{1} u^{5} \mathrm{~d} x\right)^{1 / 3}+g\left(\int_{0}^{1} v^{5} \mathrm{~d} x\right)^{1 / 3}\right] \\
& +\frac{\alpha_{23}}{5 \epsilon} \xi^{2} \int_{0}^{1} u^{5} \mathrm{~d} x+\frac{3 \alpha_{23}}{10 \epsilon} \xi^{2} \int_{0}^{1} v^{5} \mathrm{~d} x+\frac{\alpha_{23} \epsilon \xi}{2}\left(b d_{1}^{-1}+g d_{1}^{-1}+\xi\right) \int_{0}^{1} v w_{t}^{2} \mathrm{~d} x, \\
& \alpha_{31} \xi \int_{0}^{1} w u_{t} h \mathrm{~d} x \leq \frac{\alpha_{31} s \xi}{2 \epsilon} M_{1}^{2 / 3} d_{1}^{-1} d_{2}^{-(4 / 3)}\left(\int_{0}^{1} w^{5} \mathrm{~d} x\right)^{1 / 3}+\frac{\alpha_{31} e_{5}}{5 \epsilon} \xi^{2} \int_{0}^{1} u^{5} \mathrm{~d} x \\
& +\frac{\alpha_{31} \xi^{2}}{2 \epsilon}\left(r+\frac{3}{5} e\right) \int_{0}^{1} w^{5} \mathrm{~d} x+\frac{\alpha_{31} \epsilon \xi}{2}\left(s d_{1}^{-1}+r \xi+e \xi\right) \int_{0}^{1} w u_{t}^{2} \mathrm{~d} x, \\
& \alpha_{32} \xi \int_{0}^{1} w v_{t} h \mathrm{~d} x \leq \frac{\alpha_{32} s \xi}{2 \epsilon} M_{1}^{2 / 3} d_{1}^{-1} d_{2}^{-(4 / 3)}\left(\int_{0}^{1} w^{5} \mathrm{~d} x\right)^{1 / 3}+\frac{\alpha_{32} e}{5 \epsilon} \xi^{2} \int_{0}^{1} u^{5} \mathrm{~d} x \\
& +\frac{\alpha_{32} \xi^{2}}{2 \epsilon}\left(r+\frac{3}{5} e\right) \int_{0}^{1} w^{5} \mathrm{~d} x+\frac{\alpha_{32} \epsilon \xi}{2}\left(s d_{1}^{-1}+r \xi+e \xi\right) \int_{0}^{1} w v_{t}^{2} \mathrm{~d} x .
\end{aligned}
$$


Therefore,

$$
\begin{aligned}
\int_{0}^{1}(1 & \left.+p_{11} \xi\right) u_{t} f \mathrm{~d} x+\xi \int_{0}^{1}\left(1+p_{22}\right) v_{t} k \mathrm{~d} x+\int_{0}^{1}\left(\eta+p_{33} \xi\right) w_{t} h \mathrm{~d} x \\
& +\xi \int_{0}^{1} p_{12} v_{t} f \mathrm{~d} x+\xi \int_{0}^{1} p_{13} w_{t} f \mathrm{~d} x+\xi \int_{0}^{1} p_{21} u_{t} k \mathrm{~d} x \\
& +\xi \int_{0}^{1} p_{23} w_{t} k \mathrm{~d} x+\xi \int_{0}^{1} p_{31} u_{t} h \mathrm{~d} x+\xi \int_{0}^{1} p_{32} v_{t} h \mathrm{~d} x \\
\leq & \lambda \epsilon \int_{0}^{1}(u+v+w)\left(u_{t}^{2}+v_{t}^{2}+w_{t}^{2}\right) \mathrm{d} x+\frac{C_{8}}{\epsilon} M_{0} d_{1}^{-1} d_{2}^{-2}(1+\xi+\eta) \\
& +\frac{C_{9}}{\epsilon} M_{1}^{2 / 3} d_{2}^{-(4 / 3)} \xi\left(1+d_{1}^{-1}+\xi+\eta\right)\left[\int_{0}^{1}\left(u^{5}+v^{5}+w^{5}\right) \mathrm{d} x\right]^{1 / 3} \\
& +\frac{C_{10}}{\epsilon} \xi^{2} \int_{0}^{1}\left(u^{5}+v^{5}+w^{5}\right) \mathrm{d} x,
\end{aligned}
$$

where $\lambda$ is a positive constant. Choose a small enough positive number $\varepsilon=\varepsilon\left(d_{i}, \alpha_{i j}, i, j=1,2,3, a, b, g, s\right.$, $r, e)$ such that $\lambda \varepsilon<C_{4}$. Combining (74) and (76), one can obtain

$$
\begin{aligned}
\frac{1}{2} \bar{y}^{\prime}(t) \leq & -\frac{1}{2} \int_{0}^{1} P_{x x}^{2} \mathrm{~d} x-\frac{\xi}{2} \int_{0}^{1} Q_{x x}^{2} \mathrm{~d} x-\frac{\eta}{2} \int_{0}^{1} R_{x x}^{2} \mathrm{~d} x \\
& +B_{1} K_{2} d_{1}^{-1} d_{2}^{-1}+B_{2} K_{3} d_{1}^{-(4 / 3)} z^{1 / 3} \\
& +B_{3} K_{4} d_{1}^{-(2 / 3)} z^{2 / 3}+B_{4} K_{5} z
\end{aligned}
$$

where $\quad z=\int_{0}^{1}\left(u^{5}+v^{5}+w^{5}\right) \mathrm{d} x, K_{2}=(1+\xi+\eta)$ $\left(M_{0}+M_{1} d_{1}^{-1} d_{2}^{-1}\right), K_{3}=M_{1}^{2 / 3} \xi\left(1+d_{1}^{-1}+\xi+\eta\right)$, $K_{4}=M_{1}^{1 / 2} \xi^{2}(1+\eta)$, and $K_{5}=\xi^{2}$. Clearly, $P \geq \alpha_{11}$ $\xi u^{2}, Q \geq \alpha_{22} \xi v^{2}$, and $R \geq \alpha_{33} \xi w^{2}$. It follows from (49) that $|P|_{5 / 2}^{5 / 2} \leq C\left(\left|P_{x}\right|_{2}|P|_{1}^{3 / 2}+|P|_{1}^{5 / 2}\right)$, and

$$
\begin{aligned}
z & \leq B_{5} \xi^{-(5 / 2)} \int_{0}^{1}\left(P^{5 / 2}+Q^{5 / 2}+R^{5 / 2}\right) \mathrm{d} x \\
& \leq B_{6} \xi^{-(5 / 2)} K_{1}^{3 / 2} d_{1}^{-(3 / 2)} \bar{y}^{1 / 2}+B_{6} \xi^{-(5 / 2)} K_{1}^{5 / 2} d_{1}^{-(5 / 2)}, \\
z^{1 / 3} & \leq B_{7} \xi^{-(5 / 6)} K_{1}^{1 / 2} d_{1}^{-(1 / 2)} \bar{y}^{1 / 6}+B_{7} \xi^{-(5 / 6)} K_{1}^{5 / 6} d_{1}^{-(5 / 6)}, \\
z^{2 / 3} & \leq B_{8} \xi^{-(5 / 3)} K_{1} d_{1}^{-1} \bar{y}^{1 / 3}+B_{8} \xi^{-(5 / 3)} K_{1}^{5 / 3} d_{1}^{-(5 / 3)} .
\end{aligned}
$$

Moreover, it follows by (50) that $\left|P_{x}\right|_{2}^{10 / 3} \leq$ $C\left(\left|P_{x x}\right|_{2}^{2}|P|_{1}^{4 / 3}+|P|_{1}^{10 / 3}\right) \leq B_{9} K_{1}^{4 / 3} d_{1}^{-(4 / 3)}\left(\left|P_{x x}\right|_{2}^{2}+K_{1}^{2}\right.$ $\left.d_{1}^{-2}\right)$. So,

$$
\begin{aligned}
& -\frac{\xi}{2} \int_{0}^{1} P_{x x}^{2} \mathrm{~d} x-\frac{1}{2} \int_{0}^{1} Q_{x x}^{2} \mathrm{~d} x-\frac{\eta}{2} \int_{0}^{1} R_{x x}^{2} \mathrm{~d} x \\
& \leq-B_{10} \min \{1, \xi, \eta\} K_{1}^{-(4 / 3)} d_{1}^{4 / 3} \bar{y}^{5 / 3}+(1+\xi+\eta) K_{1}^{2} d_{1}^{-2} .
\end{aligned}
$$

Substituting the above inequality into (80) and then multiplying it by $d_{2}^{2}$, we have

$$
\begin{aligned}
\frac{1}{2} y^{\prime}(t) \leq & -A_{1} \min \{1, \xi, \eta\} K_{1}^{-(4 / 3)} d_{2}^{4 / 3} y^{5 / 3} \\
& +A_{2} \xi^{-(5 / 6)} K_{1}^{1 / 2} K_{3} d_{2}^{-(1 / 6)} y^{1 / 6} \\
& +A_{3} \xi^{-(5 / 3)} K_{1} K_{4} d_{2}^{-(1 / 3)} y^{1 / 3} \\
& +A_{4} \xi^{-(5 / 2)} K_{1}^{3 / 2} K_{5} d_{2}^{-(1 / 2)} y^{1 / 2} \\
& +A_{5}\left[K_{1}^{2}(1+\xi+\eta)+K_{2} \xi\right. \\
& +K_{1}^{5 / 6} K_{3} \xi^{-(5 / 6)} d_{2}^{-(1 / 6)}+K_{1}^{5 / 3} K_{4} \xi^{-(5 / 3)} d_{2}^{-(1 / 2)} \\
& \left.+K_{1}^{5 / 2} K_{5} \xi^{-(5 / 2)} d_{2}^{-(1 / 2)}\right]
\end{aligned}
$$

where $\quad y=\int_{0}^{1}\left[\left(d_{2} P_{x}\right)^{2}+\left(d_{2} Q_{x}\right)^{2}+\left(d_{2} R_{x}\right)^{2}\right] \mathrm{d} x$. Iinequality (77) implies that there exist $\widetilde{\tau}_{2}>0$ and a positive constant $\widetilde{M}_{2}$ depending only on $a, b, g, s, r, e, d_{1}, d_{2}, d_{3}, \alpha_{i j}(i, j=1,2,3)$ such that

$$
\int_{0}^{1}\left(d_{2} P_{x}\right)^{2} \mathrm{~d} x, \int_{0}^{1}\left(d_{2} Q_{x}\right)^{2} \mathrm{~d} x, \int_{0}^{1}\left(d_{2} R_{x}\right)^{2} \mathrm{~d} x \leq \widetilde{M_{2}}, \quad t \geq \tilde{\tau_{2}} .
$$

In the case that $d_{1}, d_{2}, d_{3} \geq 1, d_{2} / d_{1}, d_{3} / d_{1} \in[\underline{d}, \bar{d}]$, the coefficients of inequality (83) can be estimated by some constants depending on $\underline{d}$ and $\bar{d}$ but doing not on $d_{1}, d_{2}$, and $d_{3}$. So, $\widetilde{M_{2}}$ depends on $\alpha_{i j}(i, j=$ $1,2,3), a, b, g, r, s, e, d, \bar{d}$ and is irrelevant to $d_{1}$, $d_{2}$, and $d_{3}$ when $d_{1}, d_{2}, d_{3} \geq 1, d_{2} / d_{1}, d_{3} / d_{1} \in[\underline{d}, \bar{d}]$. Since 


$$
\begin{aligned}
\left(\begin{array}{c}
u_{x} \\
v_{x} \\
w_{x}
\end{array}\right) & =J^{-1}\left(\begin{array}{c}
P_{x} \\
Q_{x} \\
R_{x}
\end{array}\right), \\
J & =\left(\begin{array}{lll}
P_{u} & P_{v} & P_{w} \\
Q_{u} & Q_{v} & Q_{w} \\
R_{u} & R_{v} & R_{w}
\end{array}\right),
\end{aligned}
$$

by $\operatorname{det} J>\xi \eta$, we have

$$
\begin{array}{r}
\left|d_{2} u_{x}\right|+\left|d_{2} v_{x}\right|+\left|d_{2} w_{x}\right| \leq L\left(\left|d_{2} P_{x}\right|+\left|d_{2} Q_{x}\right|+\left|d_{2} R_{x}\right|\right), \\
0<x<1, t>0,
\end{array}
$$

where $L$ is a constant depending only on $\xi, \eta, \alpha_{i j}(i, j=1,2,3)$. After scaling back and contacting estimates (84) and (85), there exist positive constants $\tau_{2}$ and $M_{2}$ depending on $\alpha_{i j}(i, j=$ $1,2,3), d_{i}(i=1,2,3), a, b, g, r, s, e$ such that

$$
\int_{0}^{1} u_{x}^{2} \mathrm{~d} x, \int_{0}^{1} v_{x}^{2} \mathrm{~d} x, \int_{0}^{1} w_{x}^{2} \mathrm{~d} x \leq M_{2}, \quad t \geq \tau_{2}
$$

When $d_{1}, d_{2}, d_{3} \geq 1, d_{2} / d_{1}, d_{3} / d_{1} \in[\underline{d}, \bar{d}], M_{2}$ depends on $\underline{d}$ and $\bar{d}$ but do not on $d_{1}, d_{2}, d_{3} \geq 1$.

(2) $t \geq 0$. Modifying the dependency of the coefficients in inequalities (68)-(84), namely, replacing $M_{0}$ and $M_{1}$ with $M_{0}^{\prime}$ and $M_{1}^{\prime}$, there exists a positive constant $M_{2}^{\prime}$ depending on $\alpha_{i j}(i, j=1,2,3), d_{i}(i=1,2,3), a, b$, $g, r, s, e$ and the $W_{2}^{1}$-norm of $u_{0}, v_{0}, w_{0}$ such that

$$
\int_{0}^{1} u_{x}^{2} \mathrm{~d} x, \int_{0}^{1} v_{x}^{2} \mathrm{~d} x, \int_{0}^{1} w_{x}^{2} \mathrm{~d} x \leq M_{2}^{\prime}, \quad t \geq 0
$$

Furthermore, when $d_{i} \geq 1(i=1,2,3), \xi, \eta \in[\underline{d}, \bar{d}], M_{2}^{\prime}$ depends on $\underline{d}$, and $\bar{d}$ but do not on $d_{i}(i=1,2,3)$.

Summarizing estimates (54), (64), and (84) and the Sobolev embedding theorem, there exist positive constants $M$ and $M^{\prime}$ depending only on $\alpha_{i j}(i, j=1,2,3), d_{i}$ $(i=1,2,3), a, b, g, r, s, e$ such that (51) and (54) hold. In particular, $M$ and $M^{\prime}$ depend only on $\alpha_{i j}(i, j=1,2,3)$, $a, b, g, r, s, e, \underline{d}, \bar{d}$ but do not on $d_{i}(i=1,2,3)$ when $d_{i} \geq 1(i=1,2,3), \xi, \eta \in[\underline{d}, \bar{d}]$.

Similarly, there exists a positive constant $M^{\prime \prime}$ depending on $\alpha_{i j}(i, j=1,2,3), d_{i}(i=1,2,3), a, b, g, r, s, e$ and the initial functions $u_{0}, v_{0}$, and $w_{0}$ such that

$$
|u(\cdot, t)|_{1,2},|v(\cdot, t)|_{1,2},|w(\cdot, t)|_{1,2} \leq M^{\prime \prime}, \quad t \geq 0 .
$$

Furthermore, in the case that $d_{i} \geq 1(i=1,2,3)$, $\xi, \eta \in[\underline{d}, \bar{d}], M^{\prime \prime}$ depends only on $\underline{d}$ and $\bar{d}$ but do not on $d_{i}(i=1,2,3)$. Thus, $T=+\infty$. This completes the proof of Theorem 5 .

\section{Asymptotic Behavior}

In this section we will discuss the global asymptotic behavior of the positive equilibrium point $\widetilde{\mathbf{u}}$ for (6).

Theorem 6. Assume that all conditions in Theorem 5 are satisfied. Furthermore, if $a g<1$ and

$$
\begin{aligned}
4 \lambda \rho \tilde{u} \widetilde{v} \widetilde{w} d_{1} d_{2} d_{3}>\widetilde{u} M^{2}\left(\lambda \alpha_{23} \widetilde{v}+\rho \alpha_{32} \widetilde{w}\right)^{2}\left(d_{1}+2 \alpha_{11} M+\alpha_{12} M+\alpha_{13} M\right) \\
+\lambda \widetilde{v} M^{2}\left(\alpha_{13} \widetilde{u}+\rho \alpha_{31} \widetilde{w}\right)^{2}\left(d_{2}+\alpha_{21} M+2 \alpha_{22} M+\alpha_{23} M\right) \\
+\rho \widetilde{w} M^{2}\left(\alpha_{12} \widetilde{u}+\lambda \alpha_{21} \widetilde{v}\right)^{2}\left(d_{3}+\alpha_{31} M+\alpha_{32} M+2 \alpha_{33} M\right),
\end{aligned}
$$

where $\lambda=((2-a g) \tilde{u}+g) / g^{2}, \rho=1 / e, M$ is given by (58), then the positive equilibrium point $\widetilde{\mathbf{u}}=(\widetilde{u}, \widetilde{v}, \widetilde{w})$ for (6) is globally asymptotically stable.

Proof. Let $(u, v, w)$ be a solution of (6) with $u_{0}, v_{0}, w_{0} \geq$ $(\equiv) 0$. From the strong maximum principle for parabolic equations, we can prove that $u, v, w>0$ for any $t>0$.

Define

$$
\begin{aligned}
V(u, v, w)= & \int_{0}^{1}\left\{\left(u-\widetilde{u}-\widetilde{u} \ln \frac{u}{\widetilde{u}}\right)+\lambda\left(v-\widetilde{v}-\widetilde{v} \ln \frac{v}{\widetilde{v}}\right)\right. \\
& \left.+\rho\left(w-\widetilde{w}-\widetilde{w} \ln \frac{w}{\widetilde{w}}\right)\right\} \mathrm{d} x,
\end{aligned}
$$

where $\quad \lambda=((2-a g) \widetilde{u}+g) / g^{2}$ and $\rho=1 / e$. Obviously, $V(u, v, w)$ is nonnegative and $V(u, v, w)=0$ if and only if $u=\widetilde{u}, v=\widetilde{v}$, and $w=\widetilde{w}$. The time derivative of $V(u, v, w)$ for system (6) satisfies that

$$
\begin{aligned}
\frac{\mathrm{d} V(u, v, w)}{\mathrm{d} t}= & -\int_{0}^{1}\left\{\frac{\widetilde{u}}{u^{2}}\left(d_{1}+p_{11}\right) u_{x}^{2}+\frac{\lambda \widetilde{v}}{v^{2}}\left(d_{2}+p_{22}\right) v_{x}^{2}\right. \\
& +\frac{\rho \widetilde{w}}{w^{2}}\left(d_{3}+p_{33}\right) w_{x}^{2}+\left(\frac{\alpha_{12} \widetilde{u}}{u}+\frac{\lambda \alpha_{21} \widetilde{v}}{v}\right) u_{x} v_{x} \\
& +\left(\frac{\alpha_{13} \widetilde{u}}{u}+\frac{\rho \alpha_{31} \widetilde{w}}{w}\right) u_{x} w_{x} \\
& \left.+\left(\frac{\lambda \alpha_{23} \widetilde{v}}{v}+\frac{\rho \alpha_{32} \widetilde{w}}{w}\right) v_{x} w_{x}\right\} \mathrm{d} x \\
& -\int_{0}^{1}\left\{\frac{v}{\widetilde{u} u}(u-\widetilde{u})^{2}+\frac{b \lambda u}{\widetilde{v} v}(v-\widetilde{v})^{2}\right. \\
& +\rho r(w-\widetilde{w})^{2}-\left(\frac{g \lambda+1}{\widetilde{u}}+a\right)(u-\widetilde{u})(v-\widetilde{v}) \\
& +(1-\rho e)(u-\widetilde{u})(w-\widetilde{w})\} \mathrm{d} x .
\end{aligned}
$$


It is easy to know that the second integrand in the above equality is positive definite by the choices of $\lambda$ and $\rho$, and the first integrand in the above equality is positive semidefinite if

$$
\begin{aligned}
& 4 \lambda \rho \tilde{u} \widetilde{v} \widetilde{w}\left(d_{1}+p_{11}\right)\left(d_{2}+p_{22}\right)\left(d_{3}+p_{33}\right) \\
& +\left(\alpha_{12} \widetilde{u} v+\lambda \alpha_{21} \widetilde{v} u\right)\left(\alpha_{13} \widetilde{u} w+\rho \alpha_{31} \widetilde{w} u\right)\left(\lambda \alpha_{23} \widetilde{v} w+\rho \alpha_{32} \widetilde{w} v\right) \\
& >\rho \tilde{w}\left(\alpha_{12} \tilde{u} v+\lambda \alpha_{21} \widetilde{v} u\right)^{2}\left(d_{3}+p_{33}\right) \\
& +\lambda \widetilde{v}\left(\alpha_{13} \tilde{u} w+\rho \alpha_{31} \widetilde{w} u\right)^{2}\left(d_{2}+p_{22}\right) \\
& +\widetilde{u}\left(\lambda \alpha_{23} \tilde{v} w+\rho \alpha_{32} \widetilde{w} v\right)^{2}\left(d_{1}+p_{11}\right) .
\end{aligned}
$$

By the maximum-norm estimate in Theorem 5, condition (87) implies that (90) holds. Therefore, if the all conditions in Theorem 6 hold, there exists a positive constant $\delta$ such that

$$
\begin{aligned}
& \frac{\mathrm{d} H(u, v, w)}{\mathrm{d} t} \leq-\delta \int_{0}^{1}\left[(u-\widetilde{u})^{2}+(v-\widetilde{v})^{2}+(w-\widetilde{w})^{2}\right] \mathrm{d} x, \\
& \frac{\mathrm{d} H(u, v, w)}{\mathrm{d} t}<0, \quad(u, v, w) \neq E_{3} .
\end{aligned}
$$

Using integration by parts, the Hölder inequality and (52), one can easily verify that $\mathrm{d} / \mathrm{d} t \int_{0}^{1}\left[(u-\widetilde{u})^{2}+(v-\widetilde{v})^{2}+\right.$ $\left.(w-\widetilde{w})^{2}\right] \mathrm{d} x$ is bounded from above. Then, from Lemma 1 and (91), we have

$$
\begin{array}{r}
|u(\cdot, t)-\widetilde{u}|_{2} \longrightarrow 0, \\
|v(\cdot, t)-\widetilde{v}|_{2} \longrightarrow 0, \\
|w(\cdot, t)-\widetilde{w}|_{2} \longrightarrow 0,
\end{array}
$$

$$
t \longrightarrow \infty \text {. }
$$

It follows from the Gagliardo-Nirenberg inequality $|u(\cdot, t)|_{\infty} \leq C|u|_{1,2}^{1 / 2}|u|_{2}^{1 / 2}$ that

$$
\begin{gathered}
|u(\cdot, t)-\widetilde{u}|_{\infty} \longrightarrow 0, \\
|v(\cdot, t)-\widetilde{v}|_{\infty} \longrightarrow 0, \\
|w(\cdot, t)-\widetilde{w}|_{\infty} \longrightarrow 0,
\end{gathered}
$$

$$
t \longrightarrow \infty \text {. }
$$

Namely, $(u, v, w)$ converges uniformly to $(\widetilde{u}, \widetilde{v}, \widetilde{w})$. By the fact that $V(u, v, w)$ is decreasing for $t \geq 0$, it is obvious that $(\tilde{u}, \widetilde{v}, \widetilde{w})$ is globally asymptotically stable. So, the proof of Theorem 6 is completed.

Example 2. The following parameters satisfy all the conditions of Theorem 6:

$$
\begin{aligned}
\alpha_{11} & =2, \\
\alpha_{12} & =1, \\
\alpha_{13} & =10 \\
\alpha_{21} & =\frac{3}{2} \\
\alpha_{22} & =\frac{1}{2} \\
\alpha_{23} & =1 \\
\alpha_{31} & =10 \\
\alpha_{32} & =2, \\
\alpha_{33} & =3 \\
g & =1 \\
a & =\frac{1}{2} \\
b & =\frac{1}{2} \\
e & =1
\end{aligned}
$$$$
d_{1}, d_{2}, d_{3} \gg 1
$$

Remark 3. In model (6), if coefficients of reaction functions satisfy the conditions of Theorem 6, the diffusion matrix is positive definite and the self-diffusion coefficients are large enough; then, the model has no nonconstant positive steady state.

\section{Data Availability}

Data sharing is not applicable to this article as no datasets were generated or analysed during the current study.

\section{Conflicts of Interest}

The authors declare that there are no conflicts of interest regarding the publication of this paper. 


\section{Acknowledgments}

This research was supported by the National Natural Science Foundation of China (nos 11761063 and 11661051).

\section{References}

[1] K. G. Magnússon, "Destabilizing effect of cannibalism on a structured predator-prey system," Mathematical Biosciences, vol. 155, no. 1, pp. 61-75, 1999.

[2] F. van den Bosch, A. M. de Roos, and W. Gabriel, "Cannibalism as a life boat mechanism," Journal of Mathematical Biology, vol. 26, no. 6, pp. 619-633, 1988.

[3] M. E. Gurtin and D. S. Levine, "On populations that cannibalize their Young," SIAM Journal on Applied Mathematics, vol. 42, no. 1, pp. 94-108, 1982.

[4] O. Diekmann, R. M. Nisbet, W. S. C. Gurney, and F. van den Bosch, "Simple mathematical models for cannibalism: a critique and a new approach," Mathematical Biosciences, vol. 78, no. 1, pp. 21-46, 1986.

[5] A. Hastings, "Cycles in cannibalistic egg-larval interactions," Journal of Mathematical Biology, vol. 24, no. 6, pp. 651-666, 1987.

[6] C. Kohlmeier and W. Ebenhöh, "The stabilizing role of cannibalism in a predator-prey system," Bulletin of Mathematical Biology, vol. 57, no. 3, pp. 401-411, 1995.

[7] F. van den Bosch and W. Gabriel, "Cannibalism in an agestructured predator-prey system," Bulletin of Mathematical Biology, vol. 59, no. 3, pp. 551-567, 1997.

[8] J. D. Murray, Mathematical Biology I: An Introduction, Springer, New York, NY, USA, 3rd edition, 2003.

[9] N. Shigesada, K. Kawasaki, and E. Teramoto, "Spatial segregation of interacting species," Journal of Theoretical Biology, vol. 79, no. 1, pp. 83-99, 1979.

[10] S.-A. Shim, "Uniform boundedness and convergence of solutions to the systems with cross-diffusions dominated by selfdiffusions," Nonlinear Analysis: Real World Applications, vol. 4, no. 1, pp. 65-86, 2003.

[11] Y. S. Choi, R. Lui, and Y. Yamada, "Existence of global solutions for the Shigesada-Kawasaki-Teramoto model with strongly coupled cross-diffusion," Discrete \& Continuous Dynamical Systems- $A$, vol. 10, no. 3, pp. 719-730, 2004.

[12] P. Y. H. Pang and M. Wang, "Strategy and stationary pattern in a three-species predator-prey model," Journal of Differential Equations, vol. 200, no. 2, pp. 245-273, 2004.

[13] S. M. Fu, Z. J. Wen, and S. B. Cui, "On global solutions for the three-species food-chain model with cross-diffusion," Acta Mathematica Sinica-A, vol. 50, pp. 75-88, 2007.

[14] S. M. Fu and X. L. Yang, "Nonconstant positive steady states of a predator-prey model with cannibalism," International Journal of Information and Systems Sciences, vol. 8, pp. 250260, 2012.

[15] S. Fu, L. Zhang, and P. Hu, "Global behavior of solutions in a Lotka-Volterra predator-prey model with prey-stage structure," Nonlinear Analysis: Real World Applications, vol. 14, no. 5, pp. 2027-2045, 2013.

[16] L. Zhang and S. Fu, "Global bifurcation for a Holling-Tanner predator-prey model with prey-taxis," Nonlinear Analysis: Real World Applications, vol. 47, pp. 460-472, 2019.

[17] B. Dubey, B. Das, and J. Hussain, "A predator-prey interaction model with self and cross-diffusion," Ecological Modelling, vol. 141, no. 1-3, pp. 67-76, 2001.
[18] F. Zhang, Y. Chen, and J. Li, "Dynamical analysis of a stagestructured predator-prey model with cannibalism," Mathematical Biosciences, vol. 307, pp. 33-41, 2019.

[19] B. Buonomo and D. Lacitignola, "On the stabilizing effect of cannibalism in stage structured population models," Mathematical Biosciences and Engineering, vol. 3, pp. 717-731, 2006.

[20] K. J. Brown, P. C. Dunne, and R. A. Gardner, "A semilinear parabolic system arising in the theory of superconductivity," Journal of Differential Equations, vol. 40, no. 2, pp. 232-252, 1981.

[21] G. Simonett, "Center manifolds for quasilinear reactiondiffusion systems," Differential and Integral Equations, vol. 8, pp. 753-796, 1995.

[22] A.-K. Drangeid, "The principle of linearized stability for quasilinear parabolic evolution equations," Nonlinear Analysis: Theory, Methods \& Applications, vol. 13, no. 9, pp. 1091-1113, 1989.

[23] P. Liu, J. Shi, and Z.-A. Wang, "Pattern formation of the attraction-repulsion Keller-Segel system," Discrete \& Continuous Dynamical Systems-B, vol. 18, no. 10, pp. 2597-2625, 2013.

[24] H. Amann, "Dynamic theory of quasilinear parabolic equations-I. Abstract evolution equations," Nonlinear Analysis: Theory, Methods \& Applications, vol. 12, no. 9, pp. 895-919, 1988.

[25] H. Amann, "Dynamic theory of quasilinear parabolic equations-II. Reaction-diffusion," Differential and Integral Equations, vol. 3, pp. 13-75, 1990.

[26] H. Amann, "Dynamic theory of quasilinear parabolic systems," Mathematische Zeitschrift, vol. 202, no. 2, pp. 219-250, 1989. 